\title{
WestVirginiaUniversity
}

THE RESEARCH REPOSITORY @ WVU

Graduate Theses, Dissertations, and Problem Reports

2012

\section{Perfect Matching and Circuit Cover of Graphs}

Dong Ye

West Virginia University

Follow this and additional works at: https://researchrepository.wvu.edu/etd

\section{Recommended Citation}

Ye, Dong, "Perfect Matching and Circuit Cover of Graphs" (2012). Graduate Theses, Dissertations, and Problem Reports. 3569.

https://researchrepository.wvu.edu/etd/3569

This Dissertation is protected by copyright and/or related rights. It has been brought to you by the The Research Repository @ WVU with permission from the rights-holder(s). You are free to use this Dissertation in any way that is permitted by the copyright and related rights legislation that applies to your use. For other uses you must obtain permission from the rights-holder(s) directly, unless additional rights are indicated by a Creative Commons license in the record and/ or on the work itself. This Dissertation has been accepted for inclusion in WVU Graduate Theses, Dissertations, and Problem Reports collection by an authorized administrator of The Research Repository @ WVU.

For more information, please contact researchrepository@mail.wvu.edu. 


\section{Perfect Matching and Circuit Cover of Graphs}

A dissertation submitted in partial fulfillment of the requirements for the degree of

Doctor of Philosophy

in

Mathematics

by Dong Ye

Committee in charge:

Dr. Cun-Quan Zhang, Chair

Dr. Mark Culp

Dr. John Goldwasser

Dr. Hong-Jian Lai

Dr. Jerzy Wojciechowski

Department of Mathematics

West Virginia University, Morgantown, West Virginia

2012

Copyright @ Dong YE 2012 


\begin{abstract}
The research of my dissertation is motivated by the Circuit Double Cover Conjecture due to Szekeres and independently Seymour, that every bridgeless graph $G$ has a family of circuits which covers every edge of $G$ twice. By Fleischner's Splitting Lemma, it suffices to verify the circuit double cover conjecture for bridgeless cubic graphs.
\end{abstract}

It is well known that every edge-3-colorable cubic graph has a circuit double cover. The structures of edge-3-colorable cubic graphs have strong connections with the circuit double cover conjecture. In chapter two, we consider the structure properties of a special class of edge-3-colorable cubic graphs, which has an edge contained by a unique perfect matching. In chapter three, we prove that if a cubic graph $G$ containing a subdivision of a special class of edge-3-colorable cubic graphs, semi-Kotzig graphs, then $G$ has a circuit double cover.

Circuit extension is an approach posted by Seymour to attack the circuit double cover conjecture. But Fleischer and Kochol found counterexamples to this approach. In chapter four, we post a modified approach, called circuit extension sequence. If a cubic graph $G$ has a circuit extension sequence, then $G$ has a circuit double cover. We verify that all Fleischner's examples and Kochol's examples have a circuit extension sequence, and hence not counterexamples to our approach. Further, we prove that a circuit $C$ of a bridgeless cubic $G$ is extendable if the attachments of all odd Tuttebridges appear on $C$ consequently.

In the last chapter, we consider the properties of minimum counterexamples to the strong circuit double cover. Applying these properties, we show that if a cubic 
graph $G$ has a long circuit with at least $|V(G)|-7$ vertices, then $G$ has a circuit double cover. 


\section{Acknowledgements}

First and foremost, I am most indebted to my supervisor, Dr. Cun-Quan Zhang, for his continued encouragement and support over these last few years. It is a pleasure to work under his supervision. Without him, this dissertation could not have come about.

I would like to take this opportunity to thank Dr. Heping Zhang at Lanzhou University, who brought me to the research field of graph theory.

I would like to thank my other committee members: Dr. Mark Culp, Dr. John Goldwasser, Dr. Hong-Jian Lai, and Dr. Jerzy Wojciechowski, for their help during my studies. I also would like to thank Dr. Genghua Fan, Dr. Jing Huang, Dr. Hao Li, Dr. Rong Luo and Dr. Xiaoya Zha for their help and encouragement in my study and research.

And finally, I would like to thank the Department of Mathematics and Eberly College of Arts and Sciences at West Virginia University for providing me with an excellent study environment and support during my study as a graduate student. 


\section{DEDICATION}

To

Yingxiao and Lucas 


\section{Contents}

1 Introduction 1

1.1 Preliminaries and motivations . . . . . . . . . . . . . . . 1

1.2 Main contributions . . . . . . . . . . . . . . . . . 7

2 Edge-3-Colorable Cubic Graphs $\quad 9$

2.1 Introduction . . . . . . . . . . . . . . . . . . . 9

2.2 Cubic graphs in $\mathscr{G}_{\mathrm{UPM}} \ldots \ldots \ldots \ldots \ldots$

2.3 Kotzig graphs, 2-factor Hamiltonian graphs . . . . . . . . . . 18

3 Spanning Subgraphs and CDC 21

3.1 Introduction . . . . . . . . . . . . . . . . . . 21

3.2 Proof of Theorem $3.1 .17 \ldots \ldots \ldots$ 
3.3 Spanning subgraphs of cubic graphs . . . . . . . . . . . . . . . 34

4 Circuit Extension

4.1 Introduction . . . . . . . . . . . . . . . . . . . 38

4.2 Proof of Theorem $4.1 .8 \ldots \ldots \ldots$. . . . . . . . . . . . . . . . 41

4.3 Circuit extension and circuit double cover . . . . . . . . . . . . 43

4.4 Remarks . . . . . . . . . . . . . . . . . . . . . 48

$\begin{array}{lll}5 & \text { Circumference } & 49\end{array}$

5.1 Introduction . . . . . . . . . . . . . . . . . . . . . . 49

5.2 Faithful cover and circuit chain . . . . . . . . . . . . 51

5.3 Circuit extension and SCDCC . . . . . . . . . . . . . 57

5.4 Proof of Theorem 5.1.6 . . . . . . . . . . . . . . . . 61 


\section{Chapter 1}

\section{Introduction}

\subsection{Preliminaries and motivations}

Let $G$ be a graph with vertex set $E(G)$ and vertex set $V(G)$. A graph is connected if, for any two vertices $u$ and $v$, there is a path joining them. If $G$ is not connected, a maximal connected subgraph of $G$ is called a component of $G$. An edge $e$ of $G$ is called a bridge if the subgraph $G-e$ obtained from $G$ by deleing edge $e$ has more components than $G$. A bridgeless graph is a graph without bridges. Let $u$ be a vertex of $G$, the neighbors of $u$ in $G$ is a set of vertices which is adjacent to $u$. The set of all neighbors of $u$ in $G$ is denoted by $N_{G}(u)$. The degree of $G$, denoted by $d_{G}(u)$, is defined as $d_{G}(u):=\left|N_{G}(u)\right|$. If every vertex of $G$ has the same degree $k$, then $G$ is a $k$-regular graph. A connected 2-regular graph is called a circuit. A 3-regular graph is also called a cubic graph. If every vertex of $G$ has even degree, then $G$ is called an even graph or a cycle. A connected even graph is called an 
eulerian graph. An even-subgraph double cover of a bridgeless graph $G$ is a family of even-subgraphs which cover every edge of $G$ precisely twice. Since every even graph can be decomposed into edge-disjoint circuits, an even-subgraph double cover is equivalently to a circuit double cover.

The following is a well-known conjecture in graph theory due to Szekeres [46] and Seymour [43].

Conjecture 1.1.1 (Circuit Double Cover Conjecture). Every bridgeless graph has a circuit double cover.

The following is Fleischner's Splitting Lemma.

Lemma 1.1.2 (Fleishner's Splitting Lemma). Let $G$ be a bridgeless graph. Let u be a vertex of $G$ and $N_{G}(u)=\left\{v_{1}, \ldots, v_{k}\right\}$ with $k \geq 4$. Then there exist $v_{i}$ and $v_{j}$ such that $G-u v_{i}-u v_{j}+v_{i} v_{j}$ is bridgeless.

By Fleischner's Splitting Lemma, it suffice to prove that Circuit Double Cover Conjecture holds for all cubic graphs. So in this thesis, we only concentrate on cubic graphs.

Let $G$ be a bridgeless cubic graph, and $\mathscr{F}$ be a family of circuits of $G$. The coverage of $e$ of $\mathscr{F}$ is defined as

$$
\operatorname{Cov}_{\mathscr{F}}(e)=\mid\{C \mid C \in \mathscr{F} \text { and } e \in E(C)\} \mid
$$

Define a weight function for edges of $G$ such as

$$
\omega: E(G) \rightarrow\{1,2\}
$$


A weight function is called an eulerian weight if the total weight of every edge cut is even. A family $\mathscr{F}$ of circuits of $G$ is a faithful circuit cover of $(G, \omega)$ if $\operatorname{Cov}_{\mathscr{F}}(e)=\omega(e)$. A circuit double cover of $G$ is a faithful cover of $(G, \omega)$ with $\omega(e)=2$ for every $e \in E(G)$. However, not all bridgeless cubic graph associated with an eulerian weight has a faithful cover, for example, $\left(P_{10}, \omega_{10}\right)$ where $P_{10}$ is the Petersen graph and $\omega_{10}$ is an eulerian weight such that all edges of weight two induce a perfect matching.

A weight $\omega$ of a bridgeless cubic graph $G$ is a Hamiltonian weight if every faithful cover consists of two Hamiltonian circuit of $G$. Let $\mathscr{K}_{4}^{\Delta}$ be the family of all cubic graphs generated from $K_{4}$ by a series of $\mathrm{Y} \rightarrow \Delta$ operations, i.e., replacing a vertex by a triangle. The following conjecture provides the structure property of uniquely edge-3-colorable cubic graph. The following is the Hamiltonian weight conjecture.

Conjecture 1.1.3 (Zhang, [54], also see [37]). Let $G$ be 3-connected cubic graph $G$ having a Hamiltonian weight. Then $G \in \mathscr{K}_{4}^{\Delta}$.

The Hamiltonian weight conjecture is related to the unique edge-3-coloring conjecture.

Theorem 1.1.4 (Zhang, [52]). Let $G$ be a 3-connected Petersen-minor free cubic graph. If $G$ has a Hamiltonian weight, then $G$ is uniquely edge-3-colorable.

For the faithful cover problem, Seymour proved the following result.

Theorem 1.1.5 (Seymour, [43]). Let $G$ be a edge-3-colorable cubic graph and $\omega$ be an eulerian weight. Then $(G, \omega)$ has a faithful circuit cover.

Seymour's result is further strengthened to Petersen-minor free graphs. 
Theorem 1.1.6 (Alspach, Goddyn and Zhang, [3]). Let $G$ be a cubic graph without Petersen-minor and $\omega$ be an eulerian weight. Then $(G, \omega)$ has a faithful circuit cover.

By above theorem, every Petersen-minor free cubic graph has a circuit double cover. The circuit double cover conjecture is also verified for other special classes of cubic graphs.

Theorem 1.1.7 (Goddyn [20], Tarsi [47]). Let $G$ be a bridgeless cubic graph. If $G$ has a Hamiltonian path, then $G$ has a five even-subgraph double cover.

Let $G$ be a bridgeless cubic graph. A perfect matching of $G$ is a spanning 1-regular subgraph, and a 2-factor of $G$ is a spanning 2-regular subgraph. By Petersen's theorem that every bridgeless cubic graph has a perfect matching, then every bridgeless cubic graph has a 2-factor. The minimum number of odd circuits over all 2-factors of $G$ is called the oddness of $G$.

Theorem 1.1.8 (Huck and Kochol, [27]). Let $G$ be a bridgeless cubic graph of oddness two. Then $G$ has a five even-subgraph double cover.

Theorem 1.1.9 (Huck [26], Häggkvist and McGuinness [24]). Let G be a bridgeless cubic graph of oddness four. Then $G$ has a circuit double cover.

Let $G$ be a bridgeless cubic graph. A cubic graph $H$ is a spanning minor of $G$ if $G$ contains a subdivision of $H$ as a spanning subgraph. A Kotzig graph is a cubic graph $H$ with a 3 -edge-coloring $c: E(H) \rightarrow \mathbb{Z}_{3}$ such that $c^{-1}(\alpha) \cup c^{-1}(\beta)$ induces a Hamilton circuit of $H$ for every pair $\alpha, \beta \in \mathbb{Z}_{3}$. 
Theorem 1.1.10 (Goddyn [20], Häggkvist and Markström [22]). Let G be a bridgeless cubic graph. If $G$ contains a Kotzig graph as a spanning minor, then $G$ has a circuit double cover.

Conjecture 1.1.11 (Häggkvist and Markström, [22], Hoffmann-Ostenhof [28]). Every cyclically 4-edge-connected cubic graph contains a Kotzig graph as a spanning minor.

Let $H$ be a cubic graph with a 3 -edge-coloring $c: E(H) \rightarrow \mathbb{Z}_{3}$ and the following property

(*) edges in colors 0 and $\mu(\mu \in\{1,2\})$ induce a Hamilton circuit.

Let $F$ be the even 2-factor induced by edges in colors 1 and 2. If, for every even subgraph $S \subseteq F$, switching colors 1 and 2 of the edges of $S$ yields a new 3-edgecoloring having the property $(*)$, then each of these $2^{t-1} 3$-edge-coloring is called a semi-Kotzig coloring where $t$ is the number of components of $F$. A cubic graph $H$ with a semi-Kotzig coloring is called a semi-Kotzig graph.

Theorem 1.1.12 (Goddyn [20]). Let $G$ be a bridgeless cubic graph. If $G$ contains a semi-Kotzig graph as a spanning minor, then $G$ has a circuit double cover.

Let $G$ be a cubic graph. A spanning subgraph $H$ of $G$ is called a frame of $G$ if the contracted graph $G / H$ is an even graph. A Kotzig frame (resp. semi-Kotzig frame) is a frame such that for every non-circuit component $H_{i}$, the suppressed graph $\overline{H_{i}}$ is a Kotzig graph (resp. semi-Kotzig graph). 
Theorem 1.1.13 (Häggkvist and Markström, [22]). Let $G$ be a bridgeless cubic graph $G$. If $G$ contains a Kotzig frame with at most one non-circuit component, then $G$ has a circuit double cover.

The following is the Strong Circuit Double Cover Conjecture due to Goddyn $[20]$.

Conjecture 1.1.14 (Strong Circuit Double Cover Conjecture). Let $G$ be a cubic graph and $C$ be a given circuit. Then $G$ has a circuit double cover containing $C$.

Theorem 1.1.15 (Fleischner). Let $G$ be a bridgeless cubic graph and $C$ be a circuit. If $G-C$ has at most one vertex, then $G$ has a circuit double cover containing $C$.

Theorem 1.1.16 (Fleischner and Häggkvist [17]). Let $G$ be a bridgeless cubic graph and $C$ be a circuit. If $G-C$ is connected and has at most four vertices, then $G$ has a circuit double cover containing $C$.

Strong Circuit Double Cover Conjecture is open even for a bridgeless cubic graph $G$ and a circuit $C$ such that $G-C$ is two isolated vertices.

In order to use induction on proof of the circuit double cover conjecture and the strong circuit double cover conjecture, Seymour [44] proposed the circuit extension problem. Let $C$ be a circuit of a cubic graph $G$. A circuit $D$ is an extension of $C$ if $V(C) \subseteq V(D)$ and $E(C) \neq E(D)$. For any circuit $C$ of a bridgeless cubic graph $G$, if $C$ has an extension, then the circuit double cover conjecture is true. However, Fleishner [16] and Kochol [33] found some counterexamples.

Theorem 1.1.17 (Chan, Chudnovsky and Seymour, [7]). Let $G$ be a bridgeless cubic graph with a circuit $C$. If $G-C$ has at most one odd component, then $C$ has an extension in $G$. 


\subsection{Main contributions}

Edge-3-colorable cubic graphs service an important role in solving the circuit double cover conjecture. In Chapter 2, we consider some structure properties of edge-3colorable cubic graphs. A unique edge-3-colorable cubic graph $G$ has the properties that $G$ has a unique even 2-factor. Motivated by the unique edge-3-coloring conjecture, we consider cubic graphs which has a unique 2-factor through two given incident edges. Note that every bridgeless cubic graph has an exponential number of 2 -factors [10].

Theorem 1.2.1. Let $G$ be a 3-connected cubic graph. If $G$ has an edge contained by a unique 2-factor through two given incident edges, then $G$ is generated from $K_{4}$ by $Y \rightarrow \Delta$-operations.

Then we further characterized cubic graphs with the properties in the above theorem.

In Chapter 3, we consider spanning subgraphs and circuit double cover of graphs.

Theorem 1.2.2. Let $G$ be a cubic graph. If $G$ has a semi-Kotzig frame with at most one non-circuit component, then $G$ has a circuit double cover.

Theorems 1.1.10, 1.1.12 and 1.1.13 are corollaries of Theorem 1.2.2.

Theorem 1.2.3. Every cyclically 4-edge-connected cubic graph contains an edge-3colorable cubic graph as a spanning minor.

Theorem 1.2.4. Every 3-connected cubic graph of oddness two contains a semiKotzig frame with at most one non-circuit component. 
From Theorem 1.2.4 and Theorem 1.2.2, every bridgeless cubic graph of oddness two has a circuit double cover.

In Chapter 4, we consider circuit extension of cubic graphs. Since Seymour's circuit extension problem has counterexamples, a modified approach, so-called circuit extension process, is proposed. We verify that Fleischner's and Kochol's examples are not counterexamples to our approach. Further, we show the following result.

Theorem 1.2.5. Let $G$ be a cubic graph and $C$ be a circuit of $G$. Let $Q_{1}, \cdots, Q_{t}$ be all odd components of $G-C$. If every $Q_{i}$ has a vertex with a neighbor $v_{i}$ in $C$ such that $v_{1} v_{2} \cdots v_{t}$ is a segment of $C$, then $C$ has an extension in $G$.

Theorem 1.2.5 generalized Theorem 1.1.17.

In Chapter 5, we consider the forbidden patterns in minimum counterexamples of Strong Circuit Double Cover Conjecture. Applying these results, we have the following theorem.

Theorem 1.2.6. Let $G$ be a bridgeless cubic graph and $C$ be a circuit of $G$. If $G-C$ has at most 7 vertices, then $G$ has a circuit double cover. 


\section{Chapter 2}

\section{Edge-3-Colorable Cubic Graphs}

\section{$2.1 \quad$ Introduction}

Let $G$ be a cubic graph. If the edge set $E(G)$ of $G$ can be colored by three different colors such that any two edges sharing a common end vertex have different colors, then $G$ is edge-3-colorable. A subset $M$ of $E(G)$ is called a matching of $G$ if any two edges in $M$ are disjoint. A matching $M$ is perfect if every vertex of $G$ is incident with exactly one edge in $M$. A perfect matching is also called 1-factor. An edge3-colorable cubic graph has 3 edge disjoint perfect matchings, which is also called 1-factorization of $G$. A 2-factor of a graph $G$ is a 2-regular spanning subgraph. If $G$ is a cubic graph with a perfect matching $M$, then $G-M$ is a 2 -factor.

Theorem 2.1.1 (Petersen, [41]). Every bridgeless cubic graph has a perfect matching. 
Edge-3-colorable cubic graphs have good properties in circuit covering [54]. And also, if a cubic graph $G$ containing subdivisions of some special edge-3-colorable cubic graphs as a spanning subgraph, then $G$ also has a circuit double cover. We will discuss this in next chapter.

If an edge-3-colorable cubic graph has precisely one edge-3-coloring, then $G$ is uniquely edge-3-colorable. The smallest simple cubic graph $K_{4}$ is a uniquely edge-3colorable cubic graph. However, $K_{3,3}$ is not uniquely edge-3-colorable.

Let $\mathscr{K}_{4}^{\Delta}$ be the family of all cubic graphs generated from $K_{4}$ by a series of $\mathrm{Y} \rightarrow \Delta$ operations, i.e., replacing a vertex by a triangle. The following conjecture provides the structure property of uniquely edge-3-colorable cubic graph.

Conjecture 2.1.2 (Fiorini and Wilson, [11]). Let $G$ be a unique 3-edge-colorable cubic graph without Peterson minor. Then $G \in \mathscr{K}_{4}^{\Delta}$.

The above conjecture is known as the Unique Edge-3-Coloring Conjecture, which is verified for plane cubic graphs by Fowler [18] by using a method similar to the proof of the 4-Color Theorem.

The Unique Edge-3-Coloring Conjecture is related to the Hamilton Weight Conjecture in circuit covering of graphs. A Hamiltonian weight of a cubic graph $G$ is a mapping $\omega: E(G) \rightarrow\{1,2\}$ such that every family of circuits that covers each edge $e$ exactly $\omega(e)$ times consists of two Hamiltonian cycles. The following is the Hamiltonian Weight Conjecture.

Conjecture 2.1.3 (Zhang, [54], also see [37]). Let $G$ be 3-connected cubic graph $G$ having a Hamiltonian weight. Then $G \in \mathscr{K}_{4}^{\Delta}$. 
The following theorem provides a relation between the Hamiltonian weight conjecture and Unique edge-3-coloring conjecture.

Theorem 2.1.4 (Zhang, [52]). Let $G$ be a 3-connected Petersen-minor free cubic graph. If $G$ has a Hamiltonian weight, then $G$ is uniquely edge-3-colorable.

The Hamiltonian Weight Conjecture is verified for Petersen-minor free cubic graphs.

Theorem 2.1.5 (Lai and Zhang, [37]). Let $G$ be a 3-connected Petersen-minor free cubic graph. If $G$ has a Hamiltonian weight, then $G \in \mathscr{K}_{4}^{\Delta}$.

Let $G$ be an edge-3-colorable cubic graph, and $v$ be a vertex of $G$. The unique edge-3-coloring property of $G$ is equivalent to the following property:

(P1) for a vertex $v$ of $G$ and two edges $v v_{1}$ and $v v_{2}, G$ has a unique even 2-factor containing $v v_{1}$ and $v v_{2}$ which is a Hamiltonian circuit.

Instead of considering the unique edge-3-coloring property, it is interesting to consider the structure properties of a cubic graph $G$ has the following property:

(P2) for a vertex $v$ of $G$ and two edges $v v_{1}$ and $v v_{2}, G$ has a unique 2-factor containing $v v_{1}$ and $v v_{2}$.

In other words, a cubic graph $G$ with property (P2) has a unique perfect matching containing the edge incident with $v$ and distinct to $v v_{1}$ and $v v_{2}$. An edge $e$ of $G$ is a forcing edge if $G$ has a unique perfect matching containing it. So a cubic graph $G$ with property (P2) is equivalent to $G$ having a forcing edge.

Conjecture 2.1.2 provides some information on structure properties on 3-connected 
cubic graphs with property (P1). Here, we focus on the cubic graphs with property (P2). We present a complete characterization for cubic graphs with property (P2). Let $\mathscr{G}_{\mathrm{UE} 3 \mathrm{C}}$ and $\mathscr{G}_{\mathrm{UPM}}$ denote the family of 3-connected cubic graphs satisfying properties (P1), and (P2), respectively. Then we have the following relations.

Theorem 2.1.6. $\mathscr{G}_{U P M} \subsetneq \mathscr{K}_{4}^{\Delta} \subseteq \mathscr{G}_{U E 3 C}$.

\subsection{Cubic graphs in $\mathscr{G}_{\mathrm{UPM}}$}

A bridge of a graph is an edge whose deletion increases the number of the components.

Theorem 2.2.1 (Kotzig, [34]). Let $G$ be connected and have a unique perfect matching. Then $G$ has a bridge belonging to the perfect matching.

Theorem 2.2.2 (Plesník, [42]). Let $G$ is an $(r-1)$-edge connected graph $r$-regular with an even number of vertices. Then deleting any $r-1$ edges from $G$ results a graph with a perfect matching.

A graph $G$ is $k$-extendable if $G$ has at least $2 k+2$ vertices and any set $A$ of $k$ independent edges can be extended to a perfect matching; that is, there exists a perfect matching $M$ such that $A \subset M$. A direct corollary of Theorem 2.2.2 is following result,

Theorem 2.2.3. Every cubic bridgeless graph $G$ is 1-extendable.

The following theorem provides the structure properties for graphs in $\mathscr{G}_{\mathrm{UPM}}$. 
Theorem 2.2.4. Let $G$ be a 3-connected cubic graph. If $G$ has a forcing edge e, then:

(1) $G \in \mathscr{K}_{4}^{\Delta}$ and hence has a unique 1-factorization;

(2) the matching of $G$ containing e is a member of the unique 1-factorization.

Proof. Let $G$ be a 3 -connected cubic graph with a forcing edge $v v^{\prime}$, i.e. $G \in \mathscr{G}_{\mathrm{UPM}}$. Assume $v v^{\prime}$ is contained by a unique perfect matching $M$. Use induction on the number of vertices of $G$ to prove:

(1) $G \in \mathscr{K}_{4}^{\Delta}$;

(2) $G-M$ is a Hamiltonian circuit.

It is trivial if $G$ is $K_{4}$. So assume that (1) and (2) hold for for graphs $G^{\prime} \in \mathscr{G}_{\text {UPM }}$ with $\left|V\left(G^{\prime}\right)\right|<|V(G)|$.

Let $G^{\prime}:=G-\left\{v, v^{\prime}\right\}$. Then $G^{\prime}$ has a unique perfect matching since $v v^{\prime}$ is a forcing edge of $G$. Since $G$ is 3-connected, $G^{\prime}$ is connected. By Theorem 2.2.1, $G^{\prime}$ has a bridge $e^{\prime}=u_{1} u_{2}$. Deleting $e^{\prime}$ separates $G^{\prime}$ into two components $G_{1}^{\prime}$ and $G_{2}^{\prime}$. Assume that $u_{i} \in G_{i}^{\prime}$. Since $G$ is 3 -connected, both $v$ and $v^{\prime}$ have neighbors in $G_{i}^{\prime}$, say $v_{i}$ and $v_{i}^{\prime}$, respectively. Let $S_{i}$ be the edge set consisting of $e^{\prime}$ and edges from $\left\{v, v^{\prime}\right\}$ to $G_{i}^{\prime}(i=1,2)$. Let

$$
G_{i}:=G /\left(G-G_{i}^{\prime}\right) \text { for } i \in\{1,2\} \text {, }
$$

the graph obtained from $G$ by contracting $G-G_{i}^{\prime}$ into a vertex and deleting all loops. Denote the new vertex of $G_{i}$ by $u_{j}$ where $i \neq j$ and $i, j \in\{1,2\}$. Note that each $G_{i}$ is smaller than $G$ and the edge $u_{1} u_{2}$ is a forcing edge of $G_{i}$. Let $M_{i}$ be the perfect matching of $G_{i}$ containing $u_{1} u_{2}$. Hence $M_{i} \subset M$ and $M_{1} \cup M_{2}=M$. 
So by inductive hypothesis, $G_{i} \in \mathscr{K}_{4}^{\Delta}$ for $i \in\{1,2\}$ and $G_{i}-M_{i}$ is a Hamiltonian circuit $C_{i}$ of $G_{i}$. In $G$, let

$$
C:=C_{1}-\left\{u_{2} v_{1}, u_{2} v_{1}^{\prime}\right\}+C_{2}-\left\{u_{1} v_{2}, u_{1} v_{2}^{\prime}\right\}+v_{1} v v_{2}+v_{1}^{\prime} v v_{2}^{\prime}
$$

It is easily seen that $E(C)=E(G-M)$ and $C$ is a Hamiltonian circuit of $G-M$. So (2) follows. On the other hand, $G /\left(G_{1}^{\prime} \cup G_{2}^{\prime}\right)$ is a $K_{4}$. It follows that $G \in \mathscr{K}_{4}^{\Delta}$. This completes the proof.

Now we are ready to proof Theorem 2.1.6.

Proof of Theorem 2.1.6: Let $G$ be a 3-connected cubic graph and $v$ be a vertex of $G$. Assume $\left\{v_{1}, v_{2}, v_{3}\right\}$ be the neighborhood of $v$. Note that $v v_{1}$ and $v v_{2}$ is contained by a unique 2-factor if and only if $v v_{3}$ is contained by a unique perfect matching. Hence $\mathscr{G}_{2 \text {-factor }}=\mathscr{G}_{\mathrm{UPM}}$.

By Theorem 2.2.4, $\mathscr{G}_{\mathrm{UPM}} \subseteq \mathscr{K}_{4}{ }^{\Delta}$. The relation $\mathscr{G}_{\mathrm{UPM}} \neq \mathscr{K}_{4}{ }^{\Delta}$ can be shown by the graphs in Figure 2.1.
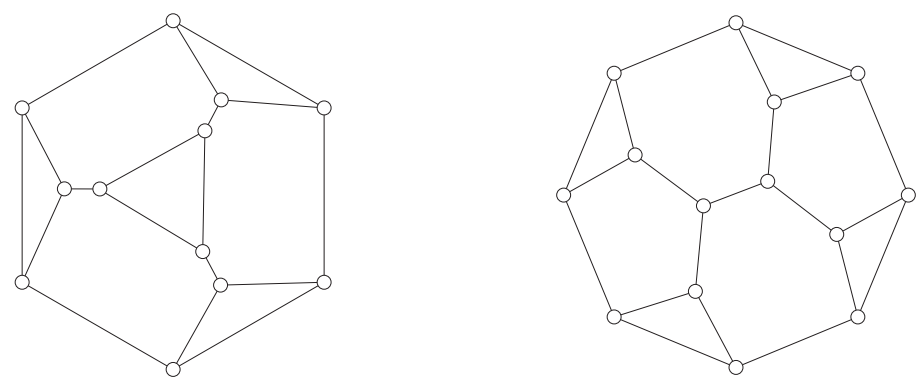

Figure 2.1. Cubic graphs in $\mathscr{K}_{4}^{\Delta}$ but not in $\mathscr{G}_{\mathrm{UPM}}$.

Let $G_{1}, G_{2} \in \mathscr{G}_{\mathrm{UPM}}$. Assume $e_{i}$ is a forcing edge of $G_{i}$ and $M_{i}$ is the perfect matching of $G_{i}$ containing $e_{i}$. Let $u_{1} v_{1} \in M_{1}$ and $e_{2}=u_{2} v_{2}$. The 2-sum of $G_{1}$ and 
$G_{2}$ is defined as

$$
G_{1} \oplus_{2} G_{2}:=G_{1}-\left\{u_{1} v_{1}\right\}+G_{2}-\left\{u_{2} v_{2}\right\}+\left\{u_{1} u_{2}, v_{1} v_{2}\right\}
$$

Clearly, $G_{1} \oplus_{2} G_{2}$ is a cubic graph and $e_{1}$ is a forcing edge of $G_{1} \oplus_{2} G_{2}$. The following result characterized all 2-connected cubic graphs with a forcing edge.

Theorem 2.2.5. Let $G$ be a 2-connected cubic graph. Then $G$ has a forcing edge if and only if $G$ is generated from graphs in $\mathscr{G}_{U P M}$ by a series of 2-sum operations.

Proof. Necessity: Let $G$ be a 2-connected cubic graph with a forcing edge $e$, and $M$ be the perfect matching containing $e$. If $G$ is 3 -connected, then $G \in \mathscr{G}_{\text {UPM }}$ and hence $G$ is generated from graphs in $\mathscr{G}_{\text {UPM }}$ by 2 -sum operation. So assume that $G$ has an two edge cut $S=\left\{u_{1} u_{2}, v_{1} v_{2}\right\}$. Deleting $S$ from $G$ separating $G$ into two subgraphs, one containing $u_{1}, v_{1}$ and the other containing $u_{2}, v_{2}$ (if necessary, relabeling the vertices). Add two new edges $u_{1} v_{1}$ and $u_{2} v_{2}$ to the two subgraphs, respectively. Then we have two 2-connected cubic graphs $G_{1}$ and $G_{2}$ such that $G_{i}$ contains $u_{i} v_{i}$.

\section{Claim: $S \subset M$.}

If not, by parity we have $S \cap M=\emptyset$. Assume that $G_{1}$ contains the edge $e$. It follows that $e$ uniquely determines a perfect matching of $M_{1}$ of $G_{1}$. For $G_{2}$, by Theorem 2.2.3, $G_{2}$ has at least two different perfect matchings $M_{2}$ and $M_{2}^{\prime}$ which contain edges incident with $u_{2}$ different from $u_{2} v_{2}$, respectively. So $M_{1} \cup M_{2}$ and $M_{1} \cup M_{2}^{\prime}$ are two different perfect matchings of $G$ containing $e$, a contradiction. Hence $S \subset M$.

If $e \notin S$, assume $e \in E\left(G_{1}\right)$. Then $G_{1}$ has a unique perfect matching $M_{1}$ 
containing $e$. If $u_{2} v_{2}$ is not a forcing edge of $G_{2}$, then there exists two different perfect matchings $M_{2}$ and $M_{2}^{\prime}$ of $G_{2}$ containing $u_{2} v_{2}$. So both $M_{1} \cup M_{2}-\left\{u_{1} v_{1}, u_{2} v_{2}\right\}+$ $\left\{u_{1} u_{2}, v_{1} v_{2}\right\}$ and $M_{1} \cup M_{2}^{\prime}-\left\{u_{1} v_{1}, u_{2} v_{2}\right\}+\left\{u_{1} u_{2}, v_{1} v_{2}\right\}$ are perfect matching of $G$ containing $e$, a contradiction. So $u_{2} v_{2}$ is a forcing edge of $G_{2}$. By induction, $G_{1}$ is generated from graphs in $\mathscr{G}_{\text {UPM }}$ by 2 -sum operations. So $G=G_{1} \oplus_{2} G_{2}$ is generated from graphs in $\mathscr{G}_{\mathrm{UPM}}$ by 2 -sum operations.

So suppose $e \in S$. Assume that $e=u_{1} u_{2}$. So $u_{1} v_{1}$ and $u_{2} v_{2}$ are forcing edges of $G_{1}$ and $G-2$, respectively. Hence $G=G_{1} \oplus_{2} G_{2}$. It follows that $G$ is generated from graphs in $\mathscr{G}_{\text {UPM }}$ by 2 -sum operations.

Sufficiency: Let $G$ be a 2-connected cubic graph generated from graphs in $\mathscr{G}_{\text {UPM }}$ by a series of 2-sum operations. Use induction on the number of 2-edge-cuts of $G$. Let $G_{0} \in \mathscr{G}_{\mathrm{UPM}}$ be the initial 3-connected cubic graph in the process generating $G$. Then $G_{0}$ has an forcing edge, say $e_{0}$. Let $S_{1}, \cdots, S_{k}$ be all 2-edge-cuts separating vertices of $G_{0}$ from vertices of $G-G_{0}$. Let $G_{1}, \cdots, G_{k}$ be all cubic graphs from $G$ by deleting all edges in $\cup_{i=1}^{k} S_{k}$ and adding new edges $e_{i}$ to every component which contains vertices of $V\left(S_{k}\right)-V\left(G_{0}\right)$. By the definition of 2-sum operation and inductive hypothesis, $e_{i}$ is a forcing edge of $G_{i}$. Note that $e_{0}$ is a forcing edge of $G_{0}$ which determines a unique perfect matching $M_{0}$ of $G_{0}$. And $\left\{e_{1}^{\prime}, e_{2}^{\prime}, \cdots, e_{k}^{\prime}\right\} \subseteq M_{0}$, where $e_{i}^{\prime}$ is an edge joining two vertices of $V\left(S_{i}\right) \cap V\left(G_{0}\right)$. So $e_{0}$ is contained by a unique perfect matching $M$ of $G$, where

$$
M=\cup_{i=0}^{k} M_{i}-\cup_{i=1}^{k} S_{i}^{\prime}+\cup_{i=1}^{k} S_{i}
$$

and $S_{i}^{\prime}=\left\{e_{i}, e_{i}^{\prime}\right\}$. This completes the proof.

Theorem 2.2.4 and Theorem 2.2.5 provide characterizations for 2-connected cubic 
graphs with a forcing edge. In the following, we consider cubic graphs with bridges.

Theorem 2.2.6. Let $G$ be a connected cubic graph with a bridge. Then $G$ has no forcing edge.

Proof. Let $G$ be a cubic graph with a bridge. So $G$ has at least two end blocks. If $G$ has no perfect matching, then it is clearly that $G$ has no forcing edge. So assume that $G$ has a perfect matching. Suppose to the contrary, that $G$ has an forcing edge $e_{0}$. Let $e=u v$ be an edge separating one of the end block which does not contain $e=u v$. Note that $G-u v$ has two components $G_{1}$ and $G_{2}$ such that $u \in G_{1}$ and $v \in G_{2}$. Let $\overline{G_{1}}$ and $\overline{G_{2}}$ be two cubic graphs from $G_{1}$ and $G_{2}$ by suppressing vertices of degree two $u$ and $v$, respectively. Assume that $G_{1}$ the end block which does not contain the forcing edge of $G$. Let $e_{u}$ and $e_{v}$ be the two edges in $E\left(\overline{G_{1}}\right)-E(G)$ and $E\left(\overline{G_{2}}\right)-E(G)$.

Since $\left|V\left(G_{1}\right)\right|=\left|V\left(\overline{G_{1}}\right)\right|+1$ and $\left|V\left(\overline{G_{1}}\right)\right| \equiv 0(\bmod 2),\left|V\left(G_{1}\right)\right|$ is odd. So $e$ belongs to all perfect matchings of $G$. Let $M_{0}$ be the perfect matching containing $e_{0}$, the forcing edge of $G$. Then $e \in M_{0}$. Let $M_{0}^{\prime}:=M_{0} \cap E\left(G_{2}\right)$. By Theorem 2.2.3, $G_{1}$ has two perfect matchings $M_{1}$ and $M_{2}$ avoiding the edge $e_{u}$. Let $M_{1}^{\prime}:=M_{1} \cup M_{0}^{\prime}$ and $M_{2}^{\prime}:=M_{2} \cup M_{0}^{\prime}$. Then both $M_{1}^{\prime}$ and $M_{2}^{\prime}$ are perfect matchings of $G$ containing $e_{0}$, a contradiction to that $e_{0}$ is a forcing edge.

Let $G \in \mathscr{K}_{4}^{\Delta}$. Note that, for any edge $e \in E(G), G-e$ has at least one triangle. So the following is a direct corollary of Theorem 2.2.4, Theorem 2.2.5 and Theorem 2.2.6.

Corollary 2.2.7. Let $G$ be a triangle-free cubic graph. Then $G$ has no forcing edge. 
For a cubic bipartite graph $G$ and a vertex $v$ of $G$, let $N(v)=\left\{v_{1}, v_{2}, v_{3}\right\}$. The uniqueness of perfect matching containing the edge $v v_{1}$ is equivalent the uniqueness of an even 2-factor containing $v v_{2}$ and $v v_{3}$. So it is also equivalent to the uniqueness of edge-3-coloring. The following result comes from Corollary 2.2.7.

Theorem 2.2.8. Let $G$ be a cubic bipartite graph. Then $G$ is not unique 3-edgecolorable.

Proof. Let $G$ be a cubic bipartite graph. Then $G$ is triangle-free. By Corollary 2.2.7, $G$ has no forcing edge. For any edge $e \in E(G), G$ has at least two different perfect matching $M_{1}$ and $M_{2}$ containing $e$. Note that $G-M_{i}$ is an even 2-factor of $G$. Let $\phi_{i}$ be an edge-3-coloring such that $\phi(e)=0$ if $e \in M_{i}$, and $\phi(e)=1$ or 2 if $e \in E\left(G-M_{i}\right)$. It follows that $\phi_{1}$ and $\phi_{2}$ are two distinct edge-3-coloring of $G$.

For a cubic bipartite graph $G$, the number of edge-3-coloring is equal to the number of perfect matchings. By a recent result of Esperet et. al [10], every cubic bipartite graph has exponentially many edge-3-colorings.

\subsection{Kotzig graphs, 2-factor Hamiltonian graphs}

Let $G$ be a graph. A 2-factor $F$ is a spanning 2-regular subgraph of $G$. A graph $G$ is 2-factor hamiltonian if every 2-factor of $G$ is a hamiltonian circuit of $G$. Examples: $K_{3,3}, K_{4}$, the Heawood graph $H$, etc.

A cubic graph $G$ is Kotzig if $G$ has an edge-3-coloring $\phi: E(G) \rightarrow \mathbb{Z}_{3}$ such that $\phi^{-1}(i) \cup \phi^{-1}(j)$ is a hamiltonian circuit where $i, j \in \mathbb{Z}_{3}$. Examples: $K_{3,3}, K_{4}$, the 
dodecahedron (20 vertices and every face is bounded by a pentagon) etc.

A cubic graph $G$ is strong-Kotzig if, for any edge-3-coloring $\phi: E(G) \rightarrow \mathbb{Z}_{3}$, $\phi^{-1}(i) \cup \phi^{-1}(j)$ is a hamiltonian circuit where $i, j \in \mathbb{Z}_{3}$. Examples: $K_{4}, K_{3,3}$ etc.

Remark. If $G \in \mathscr{G}_{\mathrm{UE} 3 \mathrm{C}}$, then $G$ has precisely three hamiltonian circuits. A cubic graph $G$ with precisely three hamiltonian circuits may not be uniquely edge-3colorable. Examples: the generalized peterson graphs $P(6 k+3,2)$ with $k \geq 2$.

Let

$$
\begin{array}{ll}
\mathscr{G}_{2 \mathrm{FH}}:=\{\text { cubic } 2 \text {-factor hamiltonian graphs }\} \\
\mathscr{G}_{\mathrm{K}}:=\{\text { Kotzig graphs }\} \\
\mathscr{G}_{\mathrm{SK}}:=\{\text { strong Kotzig graphs }\} \\
\mathscr{G}_{\mathrm{UE} 3 \mathrm{C}}:=\{\text { uniquely edge-3-colorable graphs }\} .
\end{array}
$$

We have

$$
\mathscr{G}_{2 \mathrm{FH}} \subsetneq \mathscr{G}_{\mathrm{SK}} \subsetneq \mathscr{G}_{\mathrm{K}}
$$

and

$$
\mathscr{G}_{\mathrm{UE} 3 \mathrm{C}} \subsetneq \mathscr{G}_{\mathrm{SK}}
$$

Proofs for the inequalities: $K_{3} \times P_{2} \in \mathscr{G}_{\mathrm{SK}}$ but $K_{3} \times P_{2} \notin \mathscr{G}_{2 \mathrm{FH}}$; the dodecahedron is a Kotzig graph but not a strong Kotzig; $K_{3,3} \in \mathscr{G}_{\mathrm{SK}}$ but $K_{3,3} \notin \mathscr{G}_{\mathrm{UE} 3 \mathrm{C}}$.

Proposition 2.3.1. $\mathscr{G}_{2 \mathrm{FH}} \cap \mathscr{G}_{\mathrm{UE} 3 \mathrm{C}}=\left\{K_{4}\right\}$.

Proof. Let $G \in \mathscr{G}_{2 \mathrm{FH}} \cap \mathscr{G}_{\mathrm{UE} 3 \mathrm{C}}$. Then $G$ is 1-extendable (i.e., every edge is contained in a perfect matching of $G$ ) and $G$ has precisely three perfect matchings. Note that for a cubic 1-extendable graph, it has at least $n / 4+2$ perfect matchings [38]. It follows that $n=4$. Hence $G$ is $K_{4}$. 
Let

$$
\mathscr{G}_{2 \mathrm{FHB}}:=\{\text { cubic } 2 \text {-factor Hamiltonian bipartite graphs }\} .
$$

The Heawood graph and $K_{3,3}$ are cubic 2-factor Hamiltonian bipartite graphs. By Theorem 2.2.8, $\mathscr{G}_{\mathrm{UE} 3 \mathrm{C}} \cap \mathscr{G}_{2 \mathrm{FHB}}=\emptyset$. Abreu et al. [1] conjecture that all 2-factor Hamiltonian bipartite cubic graphs are generated from $K_{3,3}$ and the Heawood graph by a series 3 -sum operations. Let $G_{1}$ and $G_{2}$ be two cubic graphs. Let $u \in V\left(G_{1}\right)$ and $v \in V\left(G_{2}\right)$. Assume $N(u)=\left\{u_{1}, u_{2}, u_{3}\right\}$ and $N(v)=\left\{v_{1}, v_{2}, v_{3}\right\}$. The 3-sum of $G_{1}$ and $G_{2}$ is defined as

$$
G_{1} \oplus_{3} G_{2}:=G_{1}-u+G_{2}-v+\left\{u_{1} v_{1}, u_{2} v_{2}, u_{3} v_{3}\right\}
$$

Conjecture 2.3.2 (Abreu et al., [1]). Every 2-factor Hamiltonian bipartite cubic graph is generated from $K_{3,3}$ and the Heawood graph by a series of 3-sum operations.

Remark. $\left\{K_{4}, K_{3,3}, H\right\}_{\oplus_{3}} \subsetneq \mathscr{G}_{\mathrm{SK}}$. Since $P(9,2) \in \mathscr{G}_{\mathrm{U} 3 \mathrm{EC}}$ and hence $P(9,2) \in \mathscr{G}_{\mathrm{SK}}$, but $P(9,2) \notin\left\{K_{4}, K_{3,3}, H\right\}_{\oplus_{3}}$. 


\section{Chapter 3}

\section{Spanning Subgraphs and CDC}

\subsection{Introduction}

Let $G$ be a graph with vertex set $V(G)$ and edge set $E(G)$. A circuit of $G$ is a connected 2-regular subgraph. A subgraph of $G$ is even if every vertex is of even degree. An even subgraph of $G$ is also called a cycle in the literatures dealing with cycle covers of graphs [31] [30] [54]. Every even graph has a circuit decomposition. A set $\mathscr{C}$ of even-subgraphs of $G$ is an even-subgraph double cover (cycle double cover) if each edge of $G$ is contained by precisely two even-subgraphs in $\mathscr{C}$. The Circuit Double Cover Conjecture was made independently by Szekeres [46] and Seymour [43].

Conjecture 3.1.1 (Szekeres [46] and Seymour [43]). Every bridgeless graph G has a circuit double cover. 
It suffices to show that the Circuit Double Cover Conjecture holds for bridgeless cubic graphs [31]. The Circuit Double Cover Conjecture has been verified for several classes of graphs; for example, cubic graphs with Hamilton paths [47] (also see [21]), cubic graphs with oddness two [27] and four [26, 24], Petersen-minor-free graphs [3].

A cubic graph $H$ is a spanning minor of a cubic graph $G$ if some subdivision of $H$ is a spanning subgraph of $G$. In [20], Goddyn showed that a cubic graph $G$ has a circuit double cover if it contains the Petersen graph as a spanning minor. Goddyn's result is further improved by Häggkvist and Markström [23] who showed that a cubic graph $G$ has a circuit double cover if it contains a 2-connected simple cubic graph with no more than 10 vertices as a spanning minor.

A Kotzig graph is a cubic graph $H$ with a 3-edge-coloring $c: E(H) \rightarrow \mathbb{Z}_{3}$ such that $c^{-1}(\alpha) \cup c^{-1}(\beta)$ induces a Hamilton circuit of $H$ for every pair $\alpha, \beta \in \mathbb{Z}_{3}$. The family of all Kotzig graphs is denoted by $\mathcal{K}$.

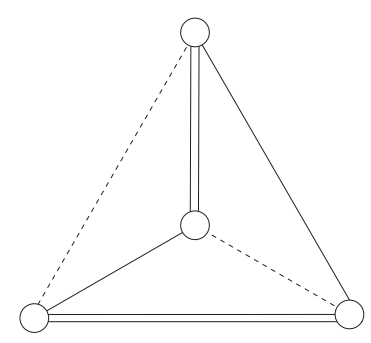

Figure 3.1: The Kotzig graph $K_{4}$.

Theorem 3.1.2 (Goddyn [20], Häggkvist and Markström [22]). If a cubic graph G contains a Kotzig graph as a spanning minor, then $G$ has a 6 -even-subgraph double cover.

By Theorem 3.1.2, any cubic graph $G$ containing some member of $\mathcal{K}$ as a spanning minor has a circuit double cover. However, we do not know yet whether every 
3-connected cubic graph contains a member of $\mathcal{K}$ as a spanning minor (Conjecture 3.1.3).

According to their observations [22, 23], Häggkvist and Markström conjectured that every 3-connected cubic graph contains a Kotzig graph as a spanning minor. In [28], Hoffmann-Ostenhof found a counterexample for this conjecture and he suggested the modified conjecture as follows.

Conjecture 3.1.3 (Häggkvist and Markström, [22], Hoffmann-Ostenhof [28]). Every cyclical 4-edge-connected cubic graph contains a Kotzig graph as a spanning minor.

Häggkvist and Markström [22] proposed another conjecture (Conjecture 3.3.1) in a more general form. We will discuss this conjecture in the last section (Remark).

One of approaches to the CDC conjecture is to find a sup-family $\mathcal{X}$ of $\mathcal{K}$ such that every bridgeless cubic graph containing a member of $\mathcal{X}$ as a spanning minor has a CDC. Following this direction of approach, Goddyn [20], Häggkvist and Markström [22] introduce some sup-families of $\mathcal{K}$, named iterated-Kotzig graphs, switchableCDC graphs and semi-Kotzig graphs. They will be defined in next subsections and their relations are shown in Figure 3.2.

\section{Iterated-Kotzig graphs}

Definition 3.1.4. An iterated-Kotzig graph $H$ is a cubic graph constructed as following [22]: Let $\mathcal{K}_{0}$ be a set of Kotzig graphs with a 3-edge-coloring $c: E(G) \rightarrow \mathbb{Z}_{3}$; A cubic graph $H \in \mathcal{K}_{i+1}$ can be constructed from a graph $H_{i} \in \mathcal{K}_{i}$ and a graph 


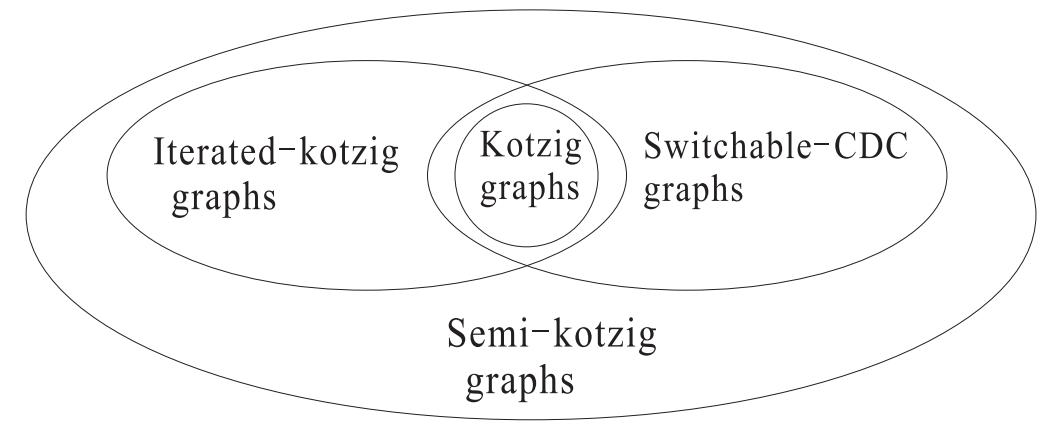

Figure 3.2: The inclusion relations for these four families: Kotzig graphs, iterated-Kotzig graphs, switchable CDC graphs, Semi-Kotzig graphs.

$H_{0} \in \mathcal{K}_{0}$ by deleting one edge colored by 0 from each of them and joining the two vertices of degree two in $H_{0}$ to the two vertices of degree two in $H_{i}$, respectively (the two new edges will be colored by 0). This operation is called 2-sum operation.

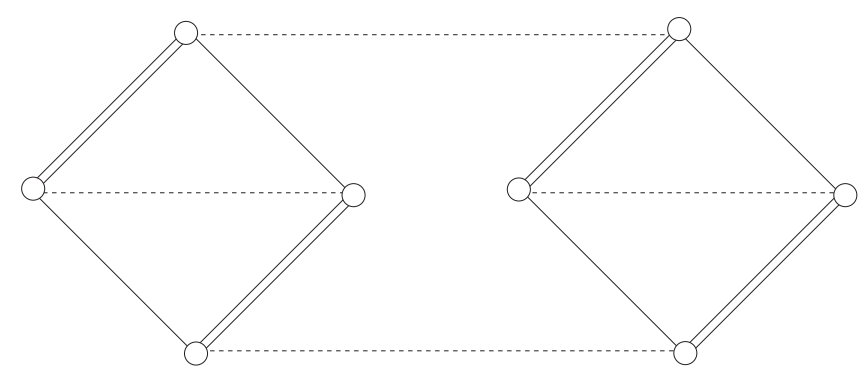

Figure 3.3: An iterated-Kotzig graph generated from two $K_{4}$ 's.

Theorem 3.1.5 (Häggkvist and Markström, [22]). If a cubic graph G contains an iterated Kotzig graph as a spanning minor, then $G$ has a 6-even-subgraph double cover. 


\section{Semi-Kotzig graphs and switchable-CDC graphs}

Definition 3.1.6. Let $G$ be a cubic graph with a 3-edge-coloring $c: E(G) \rightarrow \mathbb{Z}_{3}$ and the following property

(*) edges in colors 0 and $\mu(\mu \in\{1,2\})$ induce a Hamilton circuit.

Let $F$ be the even 2-factor induced by edges in colors 1 and 2. If, for every even subgraph $S \subseteq F$, switching colors 1 and 2 of the edges of $S$ yields a new 3-edgecoloring having the property $(*)$, then each of these $2^{t-1} 3$-edge-coloring is called a semi-Kotzig coloring where $t$ is the number of components of $F$. A cubic graph $G$ with a semi-Kotzig coloring is called a semi-Kotzig graph. If $F$ has at most two components $(t \leq 2)$, then $G$ is said to be a switchable-CDC graph (defined in [22]).

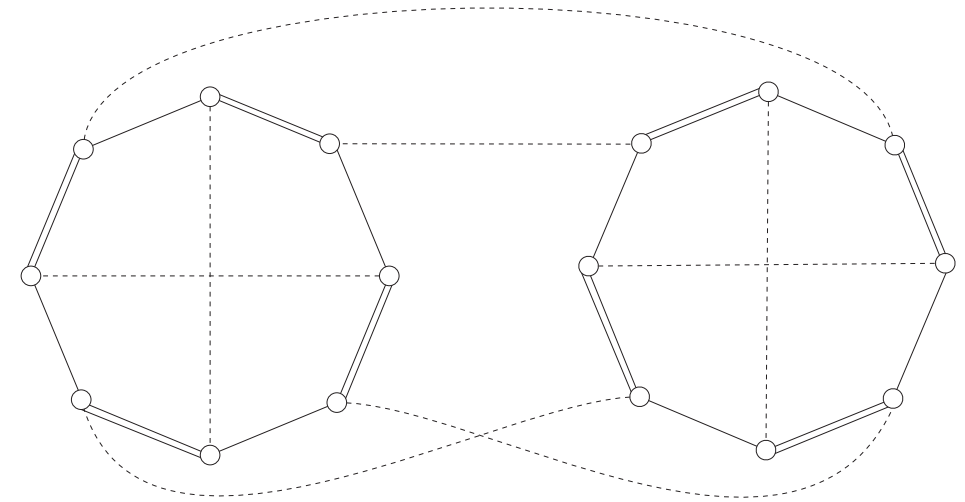

Figure 3.4: A semi-Kotzig graph.

Theorem 3.1.7 (Häggkvist and Markström, [22]). If a cubic graph $G$ contains a switchable-CDC graph as a spanning minor, then $G$ has a 6-even-subgraph double cover. 
An iterated-Kotzig graph has a semi-Kotzig coloring and hence is a semi-Kotzig graph. But a semi-Kotzig graph is not necessary an iterated-Kotzig graph. For example, the semi-Kotzig graph in Figure 3.4 is not an iterated-Kotzig graph. Hence we have the following relations (also see Figure 3.2)

$$
\begin{aligned}
& \text { Kotzig } \subset \text { Iterated-Kotzig } \subset \text { Semi-Kotzig; } \\
& \text { Kotzig } \subset \text { Switchable-CDC } \subset \text { Semi-Kotzig. }
\end{aligned}
$$

The following theorem was announced in [20] with an outline of proof.

Theorem 3.1.8 (Goddyn, [20]). If a cubic graph $G$ contains a semi-Kotzig graph as a spanning minor, then $G$ has a 6-even-subgraph double cover.

The main theorem (Theorem 3.1.17) of the paper strengthens all those early results (Theorems 3.1.2, 3.1.5, 3.1.7 and 3.1.8).

\section{Kotzig frame, semi-Kotzig frame}

A 2-factor $F$ of a cubic graph is even if every component of $F$ is of even length. If a cubic graph $G$ has an even 2-factor, then the graph $G$ has many nice properties: $G$ is 3-edge-colorable, $G$ has a circuit double cover and strong circuit double cover, etc.

The following concepts were introduced in [22] as a generalization of even 2factors.

Definition 3.1.9. Let $G$ be a cubic graph. A spanning subgraph $H$ of $G$ is called a frame of $G$ if the contracted graph $G / H$ is an even graph. 
An alternative definition of frame can be found in [22]. For a subgraph $H$ of $G$, the suppressed graph $\bar{H}$ of $H$ is the graph obtained from $H$ by suppressing all degree 2 vertices.

Definition 3.1.10. Let $G$ be a cubic graph. A frame $H$ of $G$ is called a Kotzig frame (or iterated-Kotzig frame, or switchable-CDC frame, or semi-Kotzig frame) of $G$ if, for each non-circuit component $H_{i}$ of $H$, the suppressed graph $\overline{H_{i}}$ is a Kotzig graph (or an iterated-Kotzig graph, or a switchable-CDC graph, or a semi-Kotzig graph, respectively).

Similar to the relations described in (3.1) and (3.2), we have the same relations between those frames:

$$
\begin{aligned}
& \text { Kotzig frame } \subset \text { Iterated-Kotzig frame } \subset \text { semi-Kotzig frame; } \\
& \text { Kotzig frame } \subset \text { Switchable-CDC frame } \subset \text { semi-Kotzig frame. }
\end{aligned}
$$

Theorem 3.1.11 (Häggkvist and Markström, [22]). Let $G$ be a bridgeless cubic graph $G$. If $G$ contains a Kotzig frame with at most one non-circuit component, then $G$ has a 6-even-subgraph double cover.

According to their observations, they further make the following conjecture.

Conjecture 3.1.12 (Häggkvist and Markström, [22]). Every bridgeless cubic graph with a Kotzig frame has a 6-even-subgraph double cover.

The following theorem provides a partial solution to Conjecture 3.1.12.

Theorem 3.1.13 (Zhang and Zhang, [57]). Let $G$ be a bridgeless cubic graph. If $G$ contains a Kotzig frame $H$ such that $G / H$ is a tree if parallel edges are identified as a single edge, then $G$ has a 6-even-subgraph double cover. 
In [8], Cutler and Häggkvist proved that if a cubic graph $G$ contains a frame which has two components, one of them is a subdivision of a Kotzig graph and the other is a subdivision of a semi-Kotzig graph, then $G$ has a cycle double cover.

We conjecture that the result in Conjecture 3.1.12 still holds if a Kotzig frame is replaced by a semi-Kotzig frame.

Conjecture 3.1.14. Every bridgeless cubic graph with a semi-Kotzig frame has a 6-even-subgraph double cover.

Häggkvist and Markström showed Conjecture 3.1.14 holds for iterated-kotzig frames and switchable-CDC frames with at most one non-circuit component.

Theorem 3.1.15 (Häggkvist and Markström, [22]). Let G be a bridgeless cubic graph $G$. If $G$ contains an iterated-Kotzig frame with at most one non-circuit component, then $G$ has a 6-even-subgraph double cover.

Theorem 3.1.16 (Häggkvist and Markström, [22]). Let $G$ be a bridgeless cubic graph $G$. If $G$ contains a switchable-CDC frame with at most one non-circuit component, then $G$ has a 6-even-subgraph double cover.

The following theorem is the main result of the paper, which verifies that Conjecture 3.1.14 holds if a semi-Kotzig frame has at most one non-circuit component. Since Kotzig graphs, iterated-Kotzig graphs are semi-Kotzig graphs but not vice verse, Theorems 3.1.2, 3.1.5, 3.1.7, 3.1.8, 3.1.11, 3.1.15 and 3.1.16 are corollaries of our result. The proof of the theorem will be given in Section 2 .

Theorem 3.1.17. Let $G$ be a bridgeless cubic graph. If $G$ contains a semi-Kotzig frame $H$ with at most one non-circuit component, then $G$ has a 6-even-subgraph double cover. 


\subsection{Proof of Theorem 3.1.17}

The following well-known fact will be applied in the proof of the main theorem (Theorem 3.1.17).

Lemma 3.2.1. If a cubic graph has an even 2-factor $F$, then $G$ has a 3-evensubgraph double cover $\mathscr{C}$ such that $F \in \mathscr{C}$.

Definition 3.2.2. Let $H$ be a bridgeless subgraph of a cubic graph $G$. A mapping $c: E(H) \rightarrow \mathbb{Z}_{3}$ is called a parity 3-edge-coloring of $H$ if, for each vertex $v \in H$ and each $\mu \in \mathbb{Z}_{3}$,

$$
\left|c^{-1}(\mu) \cap E(v)\right| \equiv|E(v) \cap E(H)| \quad(\bmod 2) .
$$

It is obvious that if $H$ itself is cubic, then a parity 3-edge-coloring is a proper 3-edge-coloring (traditional definition).

Preparation of the proof. Let $H_{0}$ be the component of $H$ such that $H_{0}$ is a subdivision of a semi-Kotzig graph and each $H_{i}, 1 \leq i \leq t$, be a circuit component of $H$ of even length. Let $M=E(G)-E(H)$, and $H^{*}=H-H_{0}$.

Given an initial semi-Kotzig coloring $c_{0}: E\left(\bar{H}_{0}\right) \rightarrow \mathbb{Z}_{3}$ of $\bar{H}_{0}$, then $F_{0}=c_{0}^{-1}(1) \cup$ $c_{0}^{-1}(2)$ is a 2-factor of $\bar{H}_{0}$ and $c_{0}^{-1}(0) \cup c_{0}^{-1}(\mu)$ is a Hamilton circuit of $\bar{H}_{0}$ for each $\mu \in\{1,2\}$.

The semi-Kotzig coloring $c_{0}$ of $\bar{H}_{0}$ can be considered as an edge-coloring of $H_{0}$ : each induced path is colored with the same color as its corresponding edge in $\bar{H}_{0}$ (note, this edge-coloring of $H_{0}$ is a parity 3 -edge-coloring, which may not be a proper 3-edge-coloring). 
The strategy of the proof is to show that $G$ can be covered by three subgraphs $G(0,1), G(0,2)$ and $G(1,2)$ such that each $G(\alpha, \beta)$ has a 2-even-subgraph cover which covers the edges of $M \cap E(G(\alpha, \beta))$ twice and the edges of $E(H) \cap E(G(\alpha, \beta))$ once. In order to prove this, we are going to show that the three subgraphs $G(\alpha, \beta)$ have the following properties:

(i) the suppressed cubic graph $\overline{G(\alpha, \beta)}$ is 3-edge-colorable (so that Lemma 3.2.1 can be applied to each of them);

(ii) $c_{0}^{-1}(\alpha) \cup c_{0}^{-1}(\beta) \subseteq G(\alpha, \beta)$ for each pair $\alpha, \beta \in \mathbb{Z}_{3}$;

(iii) The even subgraph $H^{*}$ has a decomposition, $H_{1}^{*}$ and $H_{2}^{*}$, each of which is an even subgraph, (here, for technical reason, let $H_{0}^{*}=\emptyset$ ), such that $H_{\alpha}^{*} \cup H_{\beta}^{*} \subseteq G(\alpha, \beta)$, for each $\{\alpha, \beta\} \subset \mathbb{Z}_{3}$;

(iv) each $e \in M=E(G)-E(H)$ is contained in precisely one member of $\{G(0,1), G(0,2), G(1,2)\}$;

(v) and most important, the subgraph $c_{0}^{-1}(\alpha) \cup c_{0}^{-1}(\beta) \cup H_{\alpha}^{*} \cup H_{\beta}^{*}$ in $G(\alpha, \beta)$ corresponds to an even 2-factor of $\overline{G(\alpha, \beta)}$.

Can we decompose $H^{*}$ and find a partition of $M=E(G)-E(H)$ to satisfy (v)? One may also notice that the initial semi-Kotzig coloring $c$ may not be appropriate. However, the color-switchability of the semi-Kotzig component $H_{0}$ may help us to achieve the goal. The properties described above in the strategy will be proved in the following claim.

We claim that $G$ has the following property: 
(*) There is a semi-Kotzig coloring $c_{0}$ of $\bar{H}_{0}$, a decomposition $\left\{H_{1}^{*}, H_{2}^{*}\right\}$ of $H^{*}$ and a partition $\left\{N_{(0,1)}, N_{(0,2)}, N_{(1,2)}\right\}$ of $M$ such that, let $C_{(\alpha, \beta)}=c_{0}^{-1}(\alpha) \cup c_{0}^{-1}(\beta)$,

(1) for each $\mu \in\{1,2\}, C_{(0, \mu)} \cup H_{\mu}^{*}$ corresponds to an even 2-factor of $\overline{G(0, \mu)}=$ $\overline{G\left[C_{(0, \mu)} \cup H_{\mu}^{*} \cup N_{(0, \mu)}\right]}$, and

(2) $C_{(1,2)} \cup H^{*}$ corresponds to an even 2-factor of $\overline{G(1,2)}=\overline{G\left[C_{(1,2)} \cup H^{*} \cup N_{(1,2)}\right]}$.

Proof of $(*)$. Let $G$ be a minimum counterexample to $(*)$. Let $c: E(H) \rightarrow \mathbb{Z}_{3}$ be a parity 3-edge-coloring of $H$ such that

(1) the restriction of $c$ on $\overline{H_{0}}$ is a semi-Kotzig coloring, and

(2) $E\left(H^{*}\right) \subseteq c^{-1}(1) \cup c^{-1}(2)$ (a set of mono-colored circuits).

Let

$$
F=c^{-1}(1) \cup c^{-1}(2)=E(H)-c^{-1}(0)
$$

Partition the matching $M$ as follows. For each edge $e=x y \in M, x y \in M_{(\alpha, \beta)}$ $\left(\alpha \leq \beta\right.$ and $\alpha, \beta \in \mathbb{Z}_{3}$ ) if $x$ is incident with two $\alpha$-colored edges and $y$ is incident with two $\beta$-colored edges. So, the matching $M$ is partitioned into six subsets:

$$
M_{(0,0)}, M_{(0,1)}, M_{(0,2)}, M_{(1,1)}, M_{(1,2)} \text { and } M_{(2,2)} \text {. }
$$

Note that this partition will be adjusted whenever the parity 3-edge-coloring is adjusted.

Claim 1. $M_{(0, \mu)} \cap G\left[V\left(H_{0}\right)\right]=\emptyset$, for each $\mu \in \mathbb{Z}_{3}$.

Suppose that $e=x y \in M_{(0, \mu)}$ where $x$ is incident with two 0-colored edges of $H_{0}$. Then, in the graph $\overline{G-e}$, the spanning subgraph $H$ retains the same property 
as itself in $G$. Since $\overline{G-e}$ is smaller than $G, \overline{G-e}$ satisfies $(*)$ : $\bar{H}_{0}$ has a semiKotzig coloring $c_{0}$ and $M-e$ has a partition $\left\{N_{(0,1)}, N_{(0,2)}, N_{(1,2)}\right\}$ and $H^{*}$ has a decomposition $\left\{H_{1}^{*}, H_{2}^{*}\right\}$. In the semi-Kotzig coloring $c_{0}$, without loss of generality, assume that $y$ subdivides a 1-colored edge of $\bar{H}_{0}$. For the graph $G$, add $e$ into $N_{(0,1)}$. This revised partition $\left\{N_{(0,1)}, N_{(0,2)}, N_{(1,2)}\right\}$ of $M$ and the resulting subgraphs $G(\alpha, \beta)$ satisfy $(*)$. This contradicts that $G$ is a counterexample.

Since $c^{-1}(0) \subseteq H_{0}$ (each component of $H-H_{0}=H^{*}$ is mono-colored by 1 or 2), for every edge $e \in M_{(0, \mu)}(\mu \in\{1,2\})$, by Claim 1, the edge $e$ has one endvertex incident with two 0 -colored edges of $H_{0}$ and its another endvertex belongs to $V\left(H-H_{0}\right)=V\left(H^{*}\right)$. That is,

$$
M_{(0,0)}=\emptyset, \text { and } M_{(0,1)} \cup M_{(0,2)} \subseteq E\left(H_{0}, H^{*}\right) .
$$

Let

$$
G^{\prime}=\overline{G-M_{(0,1)}-M_{(0,2)}} .
$$

Then $E\left(G^{\prime} / F\right) \subseteq M_{(1,1)} \cup M_{(1,2)} \cup M_{(2,2)}$.

Claim 2. The graph $G^{\prime} / F$ is acyclic.

Suppose to the contrary that $G^{\prime} / F$ contains a circuit $Q$ (including loops). In the graph $\overline{G-E(Q)}$, the spanning subgraph $H$ retains as a semi-Kotzig frame.

Then the smaller graph $\overline{G-E(Q)}$ satisfies $(*): \bar{H}_{0}$ has a semi-Kotzig coloring $c_{0}$, and $M-E(Q)$ has a a partition $\left\{N_{(0,1)}, N_{(0,2)}, N_{(1,2)}\right\}$, and $H^{*}$ has a decomposition $\left\{H_{1}^{*}, H_{2}^{*}\right\}$. So add all edges of $E(Q)$ into $N_{(1,2)}$. This revised partition $\left\{N_{(0,1)}, N_{(0,2)}, N_{(1,2)}\right\}$ of $M$ and its resulting subgraphs $G(\alpha, \beta)$ also satisfy (*) since 
$C_{(1,2)} \cup H^{*}$ corresponds to an even 2-factor of $\overline{G(1,2)}=\overline{G\left[C_{(1,2)} \cup H^{*} \cup N_{(1,2)}\right]}$. This is a contradiction. So Claim 2 follows.

By Claim 2, each component $T$ of $G^{\prime} / F$ is a tree. Along the tree $T$, we can modify the parity 3-edge-coloring $c$ of $H$ as follows:

(**) properly switch colors for some circuits in $F$ so that every edge of $T$ is incident with four same colored edges.

Note that Rule (**) is feasible by Claim 2 since $G^{\prime} / F$ is acyclic. Furthermore, under the modified parity 3-edge-coloring $c, M_{(1,2)}=\emptyset$. So

$$
M=M_{(0,1)} \cup M_{(0,2)} \cup M_{(1,1)} \cup M_{(2,2)} .
$$

The colors of all $H_{i}$ 's $(i \geq 1)$ give a decomposition $\left\{H_{1}^{*}, H_{2}^{*}\right\}$ of $H^{*}$ where $H_{\mu}^{*}$ consists of all circuits of $H^{*}$ mono-colored by $\mu$ for $\mu=1$ and 2 .

Let

$$
G^{\prime \prime}=G / H
$$

where $E\left(G^{\prime \prime}\right)=M$. Then $G^{\prime \prime}$ is even since $H$ is a frame. For a vertex $w$ of $G^{\prime \prime}$ corresponding to a component $H_{i}$ with $i \geq 1$, there is a $\mu \in\{1,2\}$ such that all edges incident with $w$ belong to $M_{(0, \mu)} \cup M_{(\mu, \mu)}$. Define

$$
N_{(0, \mu)}=M_{(0, \mu)} \cup M_{(\mu, \mu)}
$$

for each $\mu \in\{1,2\}$, and

$$
N_{(1,2)}=M_{(1,2)}=\emptyset \text {. }
$$

Hence, a vertex of $G^{\prime \prime}$ corresponding to $H_{i}$ with $i \geq 1$ either has degree in $G^{\prime \prime}\left[N_{(0, \mu)}\right]$ the same as its degree in $G^{\prime \prime}$ or has degree zero (by Rule $(* *)$ ). So every vertex of 
$G^{\prime \prime}\left[N_{(0, \mu)}\right]$ which is different from the vertex corresponding to $H_{0}$ has even degree. Since every graph has even number of odd-degree vertices, it follows that $G^{\prime \prime}\left[N_{(0, \mu)}\right]$ is an even subgraph.

For each $\mu \in\{1,2\}$, let $G(0, \mu)=N_{(0, \mu)} \cup\left(c^{-1}(0) \cup c^{-1}(\mu)\right)$. Since $G^{\prime \prime}\left[N_{(0, \mu)}\right]$ is an even subgraph of $G^{\prime \prime}$, the even subgraph $c^{-1}(0) \cup c^{-1}(\mu)$ corresponds to an even 2-factor of $G(0, \mu)$. And let $G(1,2)=F=c^{-1}(1) \cup c^{-1}(2)$ (here, $N_{(1,2)}=\emptyset$ ). So $G$ has the property $(*)$, a contradiction. This completes the proof of $(*)$.

Proof of Theorem 3.1.17. Let $G$ be a graph with a semi-Kotzig frame. Then $G$ satisfies $(*)$ and therefore is covered by three subgraphs $G(\alpha, \beta)\left(\alpha, \beta \in \mathbb{Z}_{3}\right.$ and $\alpha<\beta)$ as stated in $(*)$.

Applying Lemma 3.2.1 to the three graphs $\overline{G(\alpha, \beta)}$, each $G(0, \mu)$ has a 2-evensubgraph cover $\mathscr{C}_{(0, \mu)}$ which covers the edges of $C_{(0, \mu)} \cup H_{\mu}^{*}$ once and the edges in $N_{(0, \mu)}$ twice, and $G(1,2)$ has a 2-even-subgraph cover $\mathscr{C}_{(1,2)}$ which covers the edges of $C_{(1,2)} \cup H^{*}$ once and the edges in $N_{(1,2)}$ twice. So $\bigcup \mathscr{C}_{(\alpha, \beta)}$ is a 6-even-subgraph double cover of $G$. This completes the proof.

\subsection{Spanning subgraphs of cubic graphs}

In this section, we consider the spanning subgraphs in cubic graphs. In [22], Häggkvist and Markström proposed another conjecture which strengthens Theorems 3.1.2, 3.1.5 and 3.1.8 as follows.

Conjecture 3.3.1 (Häggkvist and Markström, [22]). If a cubic bridgeless graph contains a connected 3-edge-colorable cubic graph as a spanning minor, then G has 
a 6-even-subgraph double cover.

In fact, Conjecture 3.3.1 is equivalent to that every bridgeless cubic graph has a 6-even-subgraph double cover. It can be shown that the condition in Conjecture 3.3.1 is true for all cyclically 4-edge-connected cubic graphs.

Theorem 3.3.2. Let $G$ be a cyclically 4-edge-connected cubic graph. Then $G$ contains a connected edge-3-colorable cubic graph as a spanning minor.

Proof. Let $G$ be a cyclically 4-edge-connected cubic graph. By the Matching Polytop Theorem of Edmonds [9], $G$ has a 2-factor $F$ such that $G / F$ is 4-edge-connected. By Tutte \& Nash-Williams Theorem ([40, 49]), G/F contains two edge-disjoint spanning trees $T_{1}$ and $T_{2}$. By a theorem of Itai and Rodeh ([29]), $T_{1}$ contains a parity subgraph $P$ of $G / F$. After suppressing all degree two vertices of $G-P$, the graph $\overline{G-P}$ is 3-edge-colorable and connected since $G / F-P$ is even and $T_{2} \subset G / F-P$. So every cyclically 4-edge-connected cubic graph does contain a connected 3-edge-colorable cubic graph as a spanning minor.

Theorem 3.3.3. Let $G$ be a 3-connected cubic graph of oddness two. Then $G$ has an iterated-Kotzig frame with only one non-circuit component.

Proof. Let $G$ be a 3-connected cubic graph of oddness two. Then $G$ has a 2-factor $F$ such that $F$ has precisely two odd circuits. Consider the graph $G / F$ which is obtained from $G$ by contracting all circuits of $F$ and deleting all loops, i.e., a vertex $w$ in $G / F$ is corresponding to a circuit $C_{w}$ in $F$. So $d_{G / F}(w) \equiv\left|E\left(C_{w}\right)\right|(\bmod 2)$. Since $F$ has precisely two odd circuits, $G / F$ has only two vertices of odd degree, say $u$ and $v$. By the parity, $u$ and $v$ belong to the same one component which $G$ is 
2-connected, $G / F$ is 2-edge-connected. So it suffices to prove that $G$ is 2 -connected. In fact, we prove the following slightly stronger result.

(*) If $G$ is a 3-connected cubic graph of oddness two. Then $G$ has an iterated-Kotzig frame $H$ with only one non-circuit component $Q$ which satisfies:

(1) $Q$ is generated from $3 K_{2}, K_{4}$ and 3-prism by 2-sum operations;

(2) each generating element $K_{4}$ or 3 -prism is corresponding to a cutvertex of $G / F$.

Let $P_{1}$ and $P_{2}$ be two edge disjoint two path joining $u$ and $v$ such that $P_{1} \cap P_{2}$ as small as possible. Use induction on $\left|V\left(P_{1}\right) \cap V\left(P_{2}\right)\right|$. If $\left|V\left(P_{1}\right) \cap V\left(P_{2}\right)\right|=0$, then $P_{1} \cup P_{2}$ is a circuit of $G / F$. Let $Q$ be a subgraph of $G$ consisting of all edges of all the circuits corresponding to vertices on $P_{1} \cup P_{2}$ and all edges in $E\left(P_{1}\right) \cup E\left(P_{2}\right)$. The $\bar{H}$ is a graph obtained from $3 K_{2}$ by series of 2 -sum operations along the edges with same color (color edges of $3 K_{2}$ properly). Hence $\bar{H}$ is an iterated-Kotzig graph. So in the following, assume $V\left(P_{1}\right) \cap V\left(P_{2}\right) \neq \emptyset$.

Let $w \in V\left(P_{1}\right) \cap V\left(P_{2}\right)$. By the minimality of $P_{1} \cap P_{2}, w$ is a cut-vertex of $G$. Let $G_{1}$ and $G_{2}$ be two subgraphs of $G / F$ such that $G_{1} \cap G_{2}=w$. Let $F_{i} \subset F$ such that a circuit of $F_{i}$ is corresponding to a vertex of $G_{i}$. Let $\widehat{G_{i}}$ be the cubic graph such that $\widehat{G_{i}} / F_{i}=G_{i}$ for $i=1,2$. Then every $\widehat{G_{i}}$ is oddness-two cubic graph. By inductive hypothesis, $\widehat{G_{i}}$ satisfies $(*)$. Let $Q_{i}$ be the non-circuit component of $G_{i}$. Since $w$ is not a cut-vertex of $\widehat{G_{i}}$, in $Q_{i}, w$ is corresponding to a $3 K_{2}$. The edge used by 2 -sum operation denoted by $e_{i}(i=1,2)$. If $e_{1}$ and $e_{2}$ are crossing (the ends of $e_{1}$ and $e_{2}$ cross on the circuit $C_{w}$ ), then let $Q$ be the graph by attaching $Q_{1}$ and 
$Q_{2}$ along $C_{w}$. Then the vertex $w$ is corresponding to a $K_{4}$ in $Q$ since $e_{1}$ and $e_{2}$ are crossing. If $e_{1}$ and $e_{2}$ are not crossing, there exist and edge $e$ such that $e$ is crossing with both $e_{1}$ and $e_{2}$ since $G$ is 3 -connected. Then let $Q$ be the graph obtained by attaching $Q_{1}$ and $Q_{2}$ along $C_{w}$ plus the edge $e$. And the vertex $w$ is corresponding to a 3-prism in $Q$. This completes the proof. 


\section{Chapter 4}

\section{Circuit Extension}

\subsection{Introduction}

Let $G$ be a bridgeless graph. A subgraph of $G$ is even if every vertex is of even degree. A circuit of $G$ is a connected 2-regular graph. The following is the wellknown Circuit Double Cover Conjecture.

Conjecture 4.1.1 ([29], [43], [46], [50]). Every bridgeless graph $G$ has a family of circuits that covers every edge precisely twice.

Circuit Double Conjecture has been verified for $K_{5}$-minor-free graphs, Petersenminor-free graphs [3] and graphs with specific structures such as Hamiltonian path [47], small oddness ([27], [24], [26]) and spanning subgraphs ([20], [22], [23], [51] etc). It suffices to show that the Circuit Double Cover Conjecture holds for cubic graphs [31]. The Circuit Double Cover Conjecture is strengthened to the Strong Circuit 
Double Cover Conjecture as the following.

Conjecture 4.1.2 (Seymour, see [13] p. 237, [14], also see [20])). Let $G$ be a bridgeless cubic graph and $C$ be a circuit of $G$. Then $G$ has a circuit double cover which contains $C$.

The Strong Circuit Double Cover Conjecture is related to the Sabidussi's Compatibility Conjecture which asserts that if $T$ is an eulerian trail of an eulerian graph $G$ of minimum degree at least 4 , there exists a circuit decomposition $\mathscr{D}$ of $\mathrm{G}$ such that no transition of $T$ is contained in any element of $\mathscr{D}$. The Sabidussi's Compatibility Conjecture is equivalent to the following circuit cover version.

Conjecture 4.1.3 (Sabidussi and Fleischner [12], and Conjecture 2.4 in [4] p. 462). Let $G$ be a bridgeless cubic graph and $C$ be a dominating circuit of $G$. Then $G$ has a circuit double cover which contains $C$.

There are few partial results known for Conjecture 4.1.2 and Conjecture 4.1.3. It is well-known that 3-edge-colorable cubic graphs satisfy Conjecture 4.1.2 and Conjecture 4.1.3. The following result is obtained by Fleischner.

Theorem 4.1.4 (Fleischner [15], also see [17]). Let $G$ be a cubic graph with a circuit $C$ such that $G-V(C)$ has only one vertex. Then $G$ has a circuit double cover containing $C$.

One way to attack these conjectures is circuit extension. This idea was first proposed by Seymour (see [16] and [33]). Given a circuit $C$ of a bridgeless graph $G$, a circuit $D$ is called an extension of $C$ if $V(C) \subseteq V(D)$ and $E(D) \neq E(C)$. If $C$ has an extension in $G$, the pair $(G, C)$ is extendable. The following is a problem proposed by Seymour. 
Problem 4.1.5. Let $G$ be a bridgeless cubic graph and $C$ be a circuit. Is $(G, C)$ extendable?

If the answer of the Problem 4.1.5 is yes, then Conjectures 5.1.1, 4.1.2 and 4.1.3 will follow [54]. However, Fleischner [16] constructed a counterexample to Problem 4.1.5 and answered Seymour's problem negatively. After that, Kochol [33] constructed a infinite family of cyclic 4-edge-connected cubic graphs $G$ with circuits $C$ such that $(G, C)$ is not extendable. But it is still interesting to ask which cubic graphs have the circuit extension property.

Let $G$ be a cubic graph and $C$ be a circuit of $G$. Each component $B$ of $G-E(C)$ is called a Tutte-bridge of $C$. A vertex in $B \cap C$ is called an attachment of $B$ on $C$. A chord of $C$ is a trivial Tutte-bridge. The order of $B$ is the number of vertices in $B-V(C)$. A Tutte-bridge is odd if its order is odd.

Theorem 4.1.6 (Fleischner [15]). Let $G$ be a bridgeless cubic graph and $C$ be a circuit of $G$. If $C$ has only one non-trivial Tutte-bridge, then $(G, C)$ is extendable.

Theorem 4.1.7 (Chan, Chudnovsky and Seymour [7]). Let $G$ be a bridgeless cubic graph and $C$ be a circuit of $G$. If $C$ has only one odd Tutte-bridge, then $(G, C)$ is extendable.

In this paper, the above results are further generalized as the following, and Theorems 4.1.6 and 4.1.7 are direct corollaries of Theorem 4.1.8.

Theorem 4.1.8. Let $G$ be a bridgeless cubic graph and $C$ be a circuit of $G$. If each odd Tutte-bridge $Q_{i}(1 \leq i \leq t)$ of $C$ has an attachment $v_{i}$ such that $v_{i} v_{i+1} \in E(C)$ for $1 \leq i \leq t-1$, then $(G, C)$ is extendable. 


\subsection{Proof of Theorem 4.1.8}

Let $G$ be a cubic graph and $M$ be a set of edges of $G$. Let $G-M$ be the subgraph of $G$ obtained from $G$ by deleting all edges in $M$. The suppressed graph $\overline{G-M}$ is a graph obtained from $G-M$ by suppressing all vertices of degree two. If $M$ is a matching, $\overline{G-M}$ is a cubic graph. If $M$ has only one edge $e$, we use $G-e$ and $\overline{G-e}$ instead.

The following theorem will be used in the proof of the main theorem.

Theorem 4.2.1 (Smith's Theorem, [48]). Let $G$ be a cubic graph. Then every edge of $G$ is contained by even number of Hamiltonian circuits.

Now, we are ready to prove the main theorem.

Proof of Theorem 4.1.8. Suppose that $(G, C)$ is a minimum counterexample with $|E(G)|$ is as small as possible. Let $Q_{1}, \ldots, Q_{t}$ be all odd Tutte-bridges of $C$ such that each $Q_{i}$ has an attachment $v_{i}$ and $v_{i} v_{i+1} \in E(C)$ for $i=1, \ldots, t$.

(1) For any edge $e \in E(G) \backslash E(C), G-e$ is bridgeless.

If not, assume that $e=u v \in E(G) \backslash E(C)$ satisfies $G-e$ has a bridge $e^{\prime}=u^{\prime} v^{\prime}$. Clearly, $e$ is not a chord of $C$ and so is $e^{\prime}$. Then $G-\left\{e, e^{\prime}\right\}$ has two components $Q$ and $Q^{\prime}$. Without loss of generality, assume that $C \subseteq Q$ and $u, u^{\prime} \in Q$. By parity, $Q^{\prime}$ is not an odd Tutte-bridge. Let $G^{\prime}$ be the new cubic graph obtained from $Q$ by adding a new edge $u u^{\prime}$. Note that each $Q_{i}$ is still an odd Tutte-bridge of $C$ in $G^{\prime}$ and $v_{i} v_{i+1}$ is still an edge of $C$ in $G^{\prime}$. Since $\left|E\left(G^{\prime}\right)\right|<|E(G)|,\left(G^{\prime}, C\right)$ is extendable. Let 
$D$ be an extension of $C$ in $G^{\prime}$. If $D$ does not contain $u u^{\prime}$, then $D$ is also an extension of $C$ in $G$, a contradiction. If $D$ contains $u u^{\prime}$, then $D-u u^{\prime}+\left\{e, e^{\prime}\right\}+P_{v v^{\prime}}$ is an extension of $C$ in $G$, where $P_{v v^{\prime}}$ is a path of $Q^{\prime}$ joining $v$ and $v^{\prime}$, a contradiction. The contradiction implies that $G-e$ is bridgeless.

(2) Every non-trivial Tutte-bridge $Q$ acyclic.

Suppose to the contrary that $C$ has bridge $Q$ such that $Q$ is not isomorphic to $K_{1,3}$. If $Q$ has a circuit, let $e=u v$ be an edge on the circuit. Then the order of $\overline{Q-e}$ has the same parity as the order of $Q$. By (1), $\overline{G-e}$ is bridgeless. Note that the odd Tutte-bridges of $C$ in $\overline{G-e}$ have the same property as the odd Tutte-bridges of $C$ in $G$. Since $|E(\overline{G-e})|<|E(G)|, C$ has an extension $D$ in $\overline{G-e}$. Let $D^{\prime}$ be the corresponding circuit of $D$ in $G$, which is an extension of $C$ in $G$, a contradiction.

(3) The circuit $C$ is dominating.

By (2), every non-trivial Tutte-bridge is a tree. We are to show every non-trivial Tutte-bridge is $K_{1,3}$. Choose a leaf $v$ of $Q-V(C)$ such that $v \notin N\left(v_{i}\right)$ for all $1 \leq i \leq t$. And let $e=u v$ be an edge of $Q-V(C)$. Then $Q-e$ has two components, $Q_{1}$ and $Q_{2}$ : (i) $u, v_{i} \in V\left(Q_{1}\right)$ and $\left|V\left(\overline{Q_{1}}\right)\right| \equiv|V(Q)|(\bmod 2)$; (ii) $v \in V\left(Q_{2}\right)$ and $Q_{2}$ is a chord (joining two vertices of $N(v) \cap V(C)$ ). By (1), $\overline{G-e}$ is bridgeless and the odd Tutte-bridges of $C$ in $\overline{G-e}$ have the same property as the odd Tutte-bridges of $C$ in $G$. Note that $|E(\overline{G-e})|<|E(G)|$. Hence $C$ has an extension $D$ in $\overline{G-e}$. So the corresponding circuit $D^{\prime}$ of $D$ in $G$ is an extension of $C$ in $G$, a contradiction. The contradiction implies that $Q-V(C)$ has no edges. Since $G$ is cubic, $Q-V(C)$ is an isolated vertex. Hence $Q$ is isomorphic to $K_{1,3}$. 
(4) The final step.

By (3), $G$ has only odd Tutte-bridges $Q_{1}, \ldots, Q_{t}$ and each $Q_{i}$ has only one vertex $x_{i}$ which is not on $C$. Note that $v_{i}$ is an attachment of $Q_{i}$. So $x_{i} v_{i} \in E\left(Q_{i}\right)$. Let $M:=\left\{x_{i} v_{i} \mid i=1, \ldots, t\right\}$. Then the suppressed circuit $C^{\prime}$ of $C$ is a Hamiltonian circuit of $\overline{G-M}$, and the path $v_{1}^{-} v_{1} v_{2} \cdots v_{t} v_{t}^{+}$of $C$ is corresponding to an edge $v_{1}^{-} v_{t}^{+}$in $\overline{G-M}$. By Smith's Theorem (Theorem 4.2.1), the edge $v_{1}^{-} v_{t}^{+}$is contained in another Hamilton circuit $D^{\prime}$ which is an extension of $C^{\prime}$ in $\overline{G-M}$. Let $D$ be the circuit of $G$ which is corresponding to $D^{\prime}$. Then $D$ is an extension of $C$ in $G$, a contradiction. This completes the proof.

\subsection{Circuit extension and circuit double cover}

Circuit extension of graphs is an approach to solve the Circuit Double Cover Conjecture. The following proposition shows a strong relation between circuit extension of graphs and the Circuit Double Cover Conjecture.

Proposition 4.3.1 (Kahn, Robertson and Seymour [32], also see [7], [44], [45]). If Problem 4.1.5 is true for every circuit of all 2-connected cubic graphs, then the Circuit Double Cover Conjecture is true.

Proposition 4.3.1 can be proved by recursively applying Problem 4.1.5 as follows:

- Let $G_{0}:=G$ and $C_{0}$ be a circuit of $G$;

- Find a $C_{0}$-extension $C_{1}$ in $G$ and let $G_{1}:=\overline{G_{0}-\left(E\left(C_{0}\right)-E\left(C_{1}\right)\right)}$; 
- Find a $C_{i-1}$-extension $C_{i}$ in $G_{i-1}$ and let $G_{i}:=\overline{G_{i-1}-\left(E\left(C_{i-1}\right)-E\left(C_{i}\right)\right)}$;

- $\cdots$

- Until $G_{t-1}-\left(E\left(C_{t-2}\right)-E\left(C_{t-1}\right)\right)$ is a circuit, denoted by $C_{t}$.

This process is called a circuit-extension process with the output

$$
\left\{C_{0}, \cdots, C_{t}\right\}
$$

which is called an extension sequence of $C_{0}$.

Proposition 4.3.1 can be restated as follows.

Proposition 4.3.2. Let $G$ be a bridgeless cubic graph and $C_{0}$ be a given circuit of $G$. If, for every bridgeless subgraph $H$ of $G$, Problem 4.1 .5 is true for the suppressed cubic graph $\bar{H}$, then $G$ has a circuit double cover

$$
\left\{C_{0}, C_{0} \triangle C_{1}, \cdots, C_{t-1} \triangle C_{t}, C_{t}\right\}
$$

However, Problem 4.1.5 is not true in general: counterexamples $\left(G, C_{0}\right)$ exist (see [16, 33], also Figure 4.1 and Figure 4.2). That is, for some graphs $G$, there is a circuit $C_{0}$ which does not have an extension sequence.

Without specifying the initial circuit $C_{0}$, we suggest a modified approach as follows:

Problem 4.3.3. For a given graph $G$, is there a circuit $C_{0}$ which has an extension sequence? 


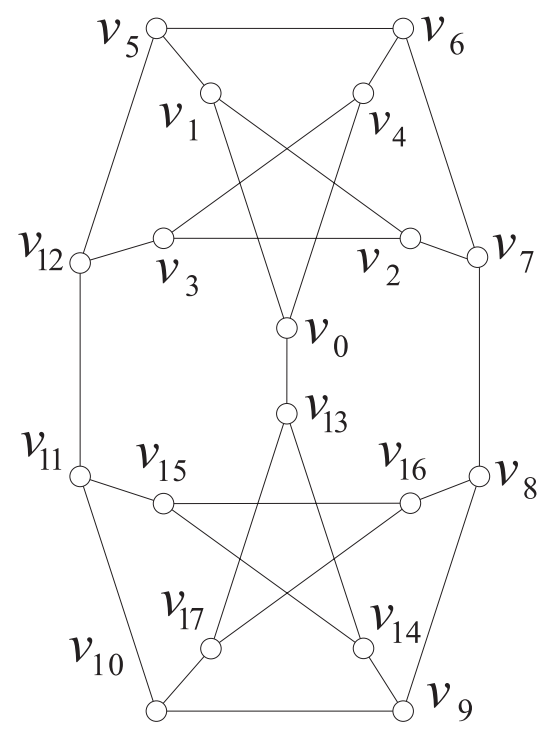

Figure 4.1: Fleischner's counterexample.

With the same argument as Proposition 4.3.2, Problem 4.3.3 implies the Circuit Double Cover Conjecture. For Problem 4.1.5, Fleischner and Kochol discovered some counterexamples. We further verify that they are not counterexamples for Problem 4.3.3 in the following two propositions.

Proposition 4.3.4. Fleischner's counterexample (see Figure 4.1) has a circuit $C_{0}$ such that the circuit-extension process can be carried out.

Proof. Let $G$ be Fleischner's counterexample as shown in Figure 4.1. Let $H$ be a bridgeless subgraph of $G$ and let $w u_{1} \cdots u_{\alpha} v$ be a path of $H$ such that $u_{1}, \ldots, u_{\alpha}$ are vertices of degree two in $H$. In $\bar{H}$, the path $w u_{1} \cdots u_{\alpha} v$ is corresponding to an edge which is denoted by $w\left(u_{1} \cdots u_{\alpha}\right) v$.

The proposition can be proved as follows, 
(1) $C_{0}=v_{0} v_{1} v_{2} v_{3} v_{4} v_{0}$, and $C_{1}=v_{0} v_{1} v_{5} v_{6} v_{7} v_{2} v_{3} v_{4} v_{0}$;

(2) $C_{1}$ has an extension $C_{2}=v_{0} v_{1} v_{5} v_{6} v_{7} v_{8} v_{16} v_{15} v_{11} v_{12} v_{3} v_{4} v_{0}$ in $G_{1}=\overline{G-v_{1} v_{2}}$

(3) $C_{2}$ has an extension $C_{3}=v_{5}\left(v_{1}\right) v_{0} v_{4} v_{6} v_{7} v_{8} v_{16} v_{15} v_{11} v_{12} v_{5}$ in $G_{2}=\overline{G_{1}-v_{7}\left(v_{2}\right) v_{3}}$;

(4) $C_{3}$ has an extension $C_{4}=v_{5}\left(v_{1}\right) v_{0} v_{13} v_{14} v_{9} v_{8} v_{16} v_{15} v_{11} v_{12} v_{5}$ in $G_{3}=\overline{G_{2}-\left\{v_{5} v_{6}, v_{12}\left(v_{3}\right) v_{4}\right\}}$;

(5) $C_{4}$ has an extension $C_{5}=v_{11} v_{10} v_{17} v_{13} v_{14} v_{9} v_{8} v_{16} v_{15} v_{11}$ in $G_{4}=\overline{G_{3}-v_{0}\left(v_{4} v_{6} v_{7}\right) v_{8}}$

(6) $C_{5}$ has an extension $C_{6}=v_{15} v_{16} v_{17} v_{10} v_{9} v_{14} v_{15}$ in $G_{5}=\overline{G_{4}-v_{13}\left(v_{0} v_{1} v_{5} v_{12}\right) v_{11}}$

(7) $C_{7}=G_{5}-\left\{v_{10}\left(v_{11}\right) v_{15}, v_{17}\left(v_{13}\right) v_{14}, v_{9}\left(v_{8}\right) v_{16}\right\}$ is a circuit.

Hence $\left\{C_{0}, C_{1}, \ldots, C_{7}\right\}$ is a desired extension sequence of $C_{0}$. This completes the proof.

Proposition 4.3.5. Kochol's counterexample (see Figure 4.2) has a circuit $C_{0}$ such that the circuit-extension process can be carried out.

An outline of the proof of this proposition is illustrated in Figure 4.2. The circuits in the extension sequence are in bold lines. The circuit in the bottom graph is a Hamilton circuit. So Smith's Theorem guarantees that the circuit-extension process can be carried out. 

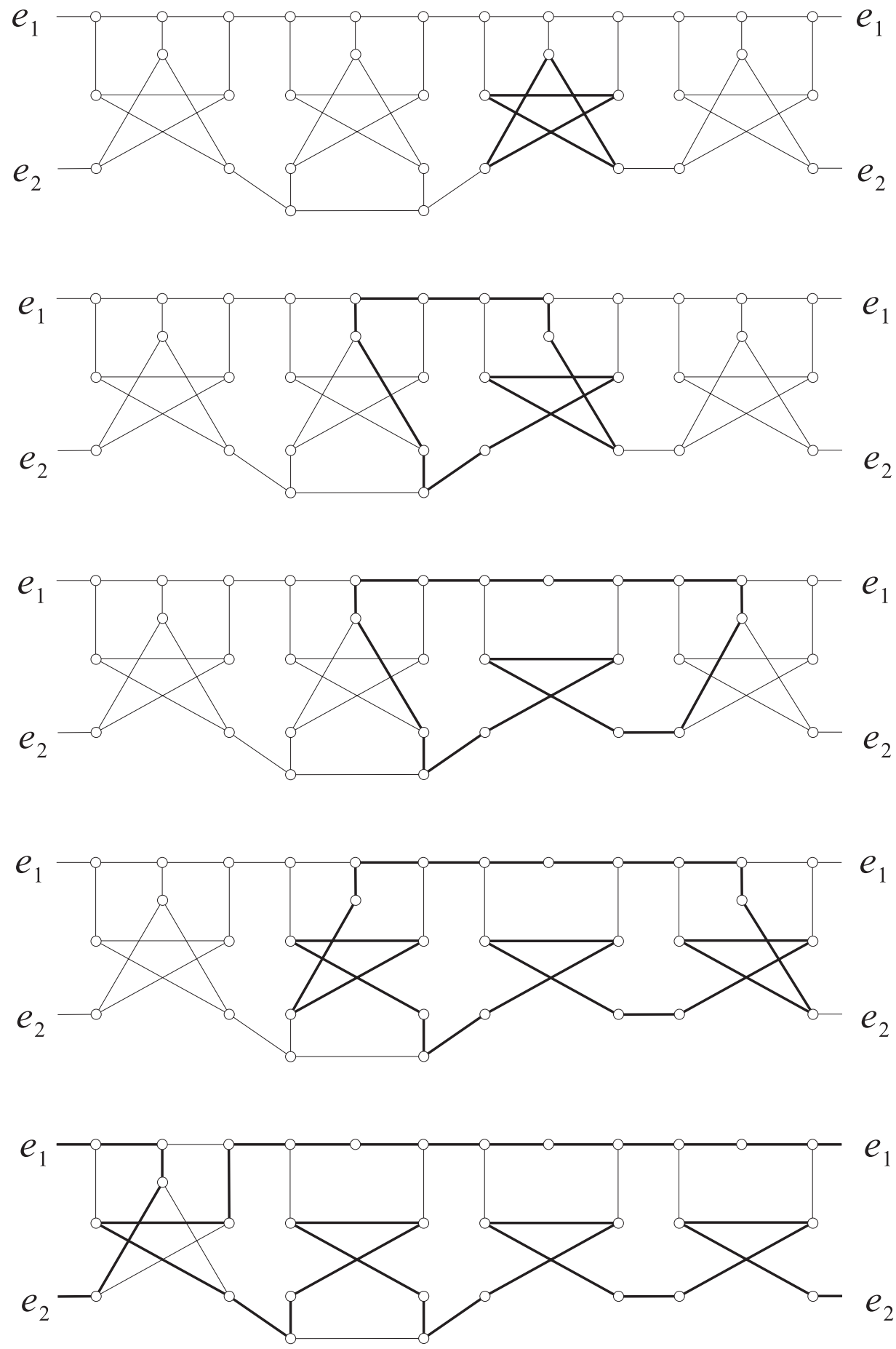

Figure 4.2: Kochol's counterexample and circuit-extension process. 


\subsection{Remarks}

Because of the existence of counterexamples to Problem 4.1.5, the circuit-extension process may not be carried out to the end: the process stops when $C_{i-1}$ has no extension in $G_{i-1}$. We propose a modification of the circuit-extension process with the following additional requirements:

(1) The initial circuit $C_{0}$ is not given, and is a shortest circuit in $G$.

(2) Among all extensions of $C_{i-1}$ in $G_{i-1}$, we choose a shortest one to be $C_{i}$.

And we conjecture that the modified circuit-extension process can be carried out to the end. 


\section{Chapter 5}

\section{Circumference}

\subsection{Introduction}

Let $G$ be a bridgless graph. A subgraph of $G$ is even if every vertex is of even degree. A circuit of $G$ is a connected 2-regular graph. The following is the well-known Circuit Double Cover Conjecture.

Conjecture 5.1.1 (CDCC, Sezekers [46], Seymour [43]). Every bridgeless graph G has a circuit double cover.

Circuit Double Conjecture has been verified for $K_{5}$-minor-free graphs, Petersenminor-free graphs and graphs with specific structures such as hamiltonian path, small oddness and spanning subgraphs.

Given a circuit $C$ of a bridgeless graph $G$, a circuit $D$ is called an extension of $C$ if $V(C) \subseteq V(D)$. A circuit $C$ is stable if it has no extension in $G$. In order to 
apply the induction to prove above conjectures, Seymour asked if any circuit $C$ of a bridgeless graph $G$ has an extension. If the answer is yes, then above conjectures follow. However, Fleischner construct a counterexample to Seymours' question. After that, Kochol constructed infinitely cyclic 4-edge-connected cubic graphs which have stable circuits. Even through there are counterexamples to Seymour's question, people proposed the following conjecture which is much stronger than CDCC.

It suffices to show that the Circuit Double Cover Conjecture holds for cubic graphs. The Circuit Double Cover Conjecture is strengthened to be the Fixed Circuit Double Cover Conjecture.

Conjecture 5.1.2 (SCDCC, Seymour, see [13] p. 237, [14], also see [20])). Let G be a bridgeless cubic graph and $C$ be a circuit of $G$. Then $G$ has a circuit double cover which contains $C$.

The Fixed Circuit Double Cover Conjecture is related to the Sabidussi's Compatibility Conjecture which asserts that if $T$ is an eulerian trail of an eulerian graph $G$ of minimum degree at least 4 , there exists a circuit decomposition $\mathscr{D}$ of $\mathrm{G}$ such that no transition of $T$ is contained in any element of $\mathscr{D}$. The Sabidussi's Compatibility Conjecture is equivalent to the following version.

Conjecture 5.1.3. Let $G$ be a bridgeless graph and $C$ be a dominating circuit of $G$. Then $G$ has a circuit double cover which contains $C$.

There are few partial results known for Conjecture 5.1.2 and Conjecture 5.1.3. It is well-known that 3-edge-colorable cubic graphs satisfy Conjecture 5.1.2 and Conjecture 5.1.3. The following result is obtained by Fleischer. 
Theorem 5.1.4 (Fleischer). Let $G$ be a cubic graph with a circuit $C$ such that $G-C$ has only one vertex. Then $G$ has a circuit double cover containing $C$.

However, Conjecture 5.1.3 remains open even for cubic graphs $G$ with a circuit $C$ such that $|G-C|=2$. Recently, Fleischner and Häggkivst obtained the following theorem.

Theorem 5.1.5 (Fleischer and Häggkivst, [17]). Let $G$ be a cubic graph and $C$ be a circuit of $G$. If $G-C$ is connected and has at most four vertices, then $G$ has a $C D C$ containing $C$.

In this paper, we show that if a cubic graph $G$ with circumference at least $n-7$, then $G$ has a circuit double cover where $n$ is the number of vertices of $G$.

Theorem 5.1.6. Let $G$ be a 2-connected cubic graph. If $G$ has a circuit $C$ such that $|G-C| \leq 7$, then $G$ has a circuit double cover containing $C$.

\subsection{Faithful cover and circuit chain}

Let $G$ be a 2-connected cubic graph. Let $\mathscr{F}$ be a family of circuits of $G$. The coverage of $e$ by $\mathscr{F}$ is defined as

$$
\operatorname{Cov}_{\mathscr{F}}(e)=\mid\{C \mid C \in \mathscr{F} \text { and } e \in E(C)\} \mid
$$

Now define a weight function for edges of $G$ such as

$$
\omega: E(G) \rightarrow\{1,2\}
$$


A weight function is called an eulerian weight if the total weight of every edge cut is even. A family $\mathscr{F}$ of circuits of $G$ is a faithful cover of $(G, \omega)$ if $\operatorname{Cov}_{\mathscr{F}}(e)=\omega(e)$. A circuit double cover of $G$ is a faithful cover of $(G, \omega)$ with $\omega(e)=2$ for every $e \in E(G)$.

In the language of faithful cover, the strong circuit double cover conjecture can be restated as the following.

Conjecture 5.2.1 (SCDCC, Seymour, see [13] p. 237, [14], also see [20])). Let G be a bridgeless cubic graph and $\omega: E(G) \rightarrow\{1,2\}$ be an eulerian weight. If $E_{\omega=1}$ induces a circuit $C$, then $(G, \omega)$ has a faithful cover.

Theorem 5.2.2 (Seymour, [43]). Let $G$ be a bridgeless cubic graph and $\omega: E(G) \rightarrow$ $\{1,2\}$ be an eulerian weight. If $G$ is 3-edge-colorable, then $(G, \omega)$ has a faithful cover.

The above theorem was generalized to Petersen-minor free graphs by Alspach and Zhang as the following.

Theorem 5.2.3 (Alspach and Zhang [5], also see [3]). Let $G$ be a bridgeless cubic graph and $\omega: E(G) \rightarrow\{1,2\}$ be an eulerian weight. If $G$ contains no subdivision of the Petersen graph, then $(G, \omega)$ has a faithful circuit cover.

Let $x$ and $y$ be two vertices of a graph $G$. A family $\mathscr{P}$ of circuits of $G$ is called a circuit chain joining $x$ and $y$ if $\mathscr{P}=\left\{C_{1}, \cdots, C_{k}\right\}$ such that:

(i) $x \in V\left(C_{1}\right)$ and $y \in V\left(C_{k}\right)$;

(ii) $V\left(C_{i}\right) \cap V\left(C_{j}\right) \neq \emptyset$ if and only if $|i-j|=1$.

The length of a circuit chain $\mathscr{P}$ is defined as the number of circuits in $\mathscr{P}$.

Lemma 5.2.4 (Lemma 3.4.1 in [56], also see [20]). Let $G$ be a bridgeless cubic graph and $\omega: E(G) \rightarrow\{1,2\}$ be an eulerian weight of $G$ and let $e=x y$ and $\omega(e)=2$. 
Assume that:

(1) $(G, \omega)$ has no faithful cover,

(2) $(\overline{G-e}, w)$ has a faithful cover.

Then the maximum circuit chain $\mathscr{P}$ joining $x$ and $y$ over all faithful covers of $(\overline{G-e}, w)$ satisfies $|\mathscr{P}| \geq 3$.

The following results give some forbidden configurations to minimum counterexamples of SCDCC.

Theorem 5.2.5. Let $(G, C)$ the a minimum counterexample to $S C D C C$. Then the girth of $G$ is at least five.

Proof. Let $C_{4}=v_{1} v_{2} v_{3} v_{4} v_{1}$. If $C=C_{4}$, then $C$ has an extension in $G$ by Theorem 4.1.8. So $\overline{G-(E(C)-E(D))}$ is smaller than $G$, and hence has a circuit double cover $\mathscr{C}$ containing $D$. So $(\mathscr{C}-\{D\}) \cup\{C, D \triangle C\}$ is a circuit double cover of $G$ containing $C$, a contradiction.

So suppose that $C_{4} \neq C$. Then $C_{4}$ has an edge with weight 2 . Without loss of generality, assume $\omega\left(v_{1} v_{4}\right)=2$.

Since $G$ is a minimum counterexample, $\overline{G-\left\{v_{1} v_{4}\right\}}$ is smaller and hence has a strong circuit double cover containing $C$. Let $\mathscr{P}$ be a maximum circuit chain over all strong circuit double covers, and let $\mathscr{C}$ be a circuit double cover containing $C$ such that $\mathscr{P} \subset \mathscr{C}$. Since the size of $C_{4}$ is four, $|\mathscr{P}| \leq 3$. By Lemma 5.2.4, $|\mathscr{P}|=3$. Let $\mathscr{P}=\left\{C_{1}, C_{2}, C_{3}\right\}$. Then the path $v_{1} v_{2} v_{3} v_{4}$ is path in some component of $C_{1} \Delta C_{2} \Delta C_{3}$. Let $C^{\prime}$ be the component containing the path $v_{1} v_{2} v_{3} v_{4}$. Let

$$
\mathscr{C}^{\prime}:=\left(\mathscr{C}-\left\{C_{1}, C_{2}, C_{3}\right\}\right) \cup\left\{\left(C_{1} \cup C_{3}\right) \Delta C_{4}, C_{2} \Delta C_{4}\right\}
$$


Then $\mathscr{C}^{\prime}$ is a circuit double cover of $G$ containing $C$.
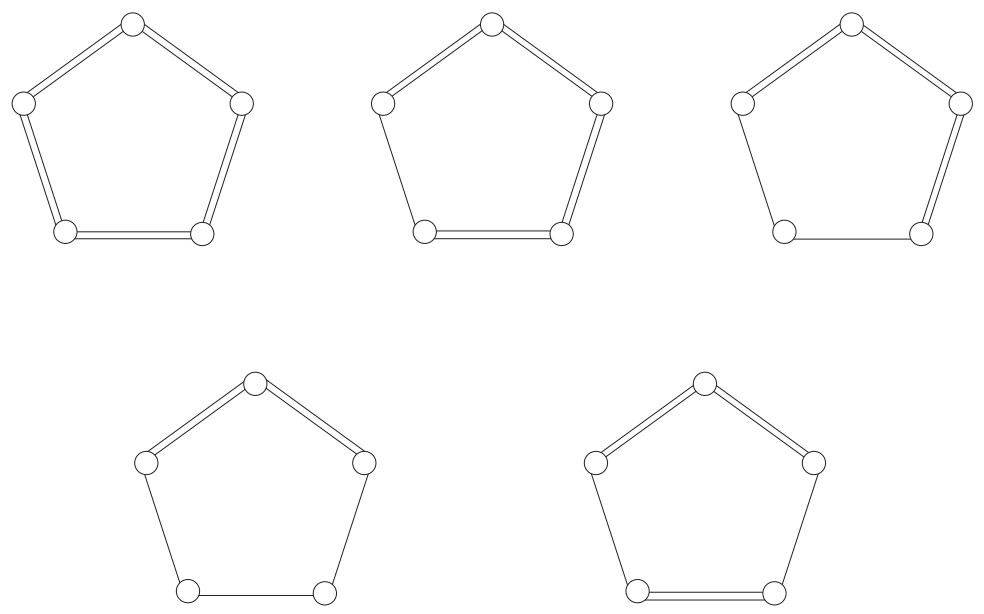

Figure 5.1. Forbidden configurations of 5-circuits in minimum counterexamples to SCDCC (Double edges with weight 2 and single edges with weight 1.)

Lemma 5.2.6. Let $(G, C)$ the a minimum counterexample to $S C D C C$. If $G$ has a circuit $C_{5} \neq C$ of size five, then $C$ contains all vertices of $C_{5}$ and there are no configurations as shown in Figure 5.1.

Proof. Suppose to the contrary that $G$ has an circuit $C_{5}$ of size five such that $C_{5} \neq C$ and $V\left(C_{5}-C\right) \neq \emptyset$. Without loss of generality, assume $C_{5}=v_{1} v_{2} v_{3} v_{4} v_{5} v_{1}$ and $v_{1} \in V\left(C_{5}-C\right)$. So $\omega\left(v_{1} v_{2}\right)=\omega\left(v_{1} v_{5}\right)=2$. Since $G$ is a minimum counterexample, $\overline{G-v_{1} v_{5}}$ is smaller and hence has a circuit double cover containing $C$. Let $\mathscr{C}$ be a such circuit double cover with a maximum circuit chain joining $v_{1}$ and $v_{5}$.

If $V\left(C_{5}\right) \cap V(C)=\emptyset$, i.e., $\omega(e)=2$ for every $e \in C_{5}$, the proof is the same as the proof of minimum counterexample to CDCC without a circuit of size five (see $[20])$. We omit the proof here. 

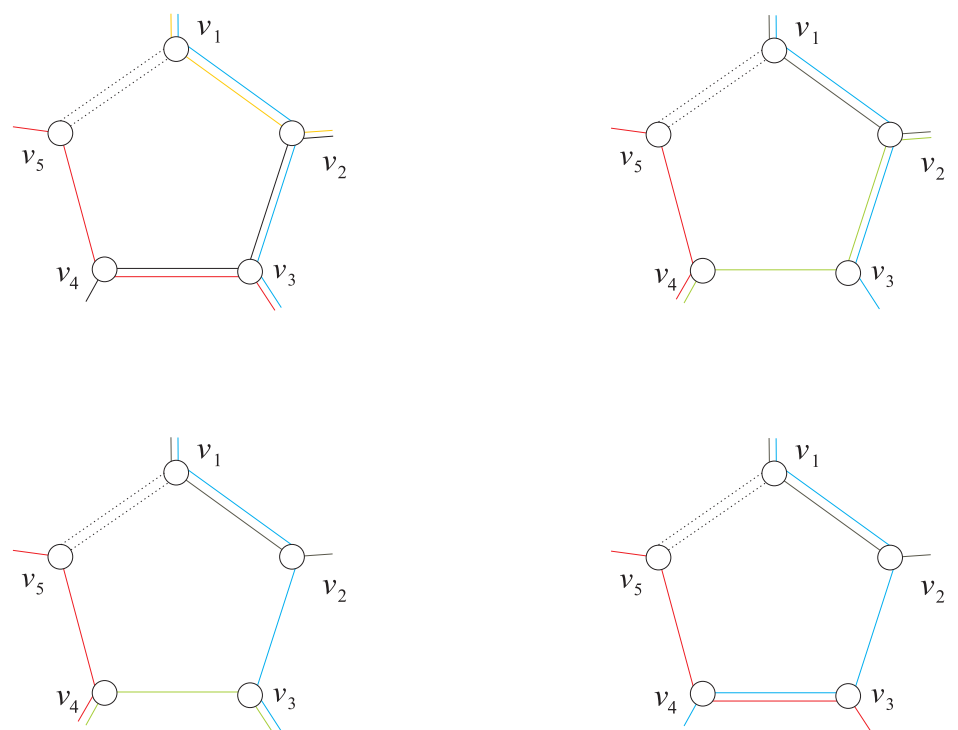

Figure 5.2. Circuit chains joining $v_{1}$ and $v_{5}$ of $C_{5}$.

So assume $C_{5}$ has at least one edge $v_{4} v_{5} \in E(C)$. If $C_{5} \cap C$ is an edge, or two disjoint edges, any circuit chain joining $v_{1}$ and $v_{5}$ has length at most two (red circuit and blue circuit), which has been illustrated in Figure 5.2 (the upper-left graph and the bottom-right graph). (Note that the pattern of the circuit chain joining $v_{1}$ and $v_{5}$ is unique.) It is a contradiction to Lemma 5.2.4.

So assume $C_{5} \cap C$ is a path of length of 2 or 3 . Then a circuit chain $\mathscr{P} \subset \mathscr{C}$ joining $v_{1}$ and $v_{5}$ has length at most 3 . By Lemma 5.2.4, $|\mathscr{P}|=3$. Since the pattern of the circuit chain $\mathscr{P}$ on $C_{5}$ is unique (see Figure 5.2: the red circuit $C_{r}$, the green circuit $C_{g}$ and the blue circuit $\left.C_{b}\right), C_{r}, C_{g}$ and $C_{b}$ are different circuits and $C_{r} \cap C_{b}=\emptyset$. So $v_{1}$ and $v_{5}$ belong to the same component of $C_{r} \Delta C_{g} \Delta C_{b}$. So let

$$
\mathscr{C}^{\prime}:=\left(\mathscr{C}-\left\{C_{r}, C_{g}, C_{b}\right\}\right) \cup\left\{\left(C_{r} \cup C_{b}\right) \Delta C_{5}, C_{g} \Delta C_{5}\right\}
$$

Then $\mathscr{C}^{\prime}$ is a circuit double cover of $G$ containing $C$, a contradiction. This completes the proof. 

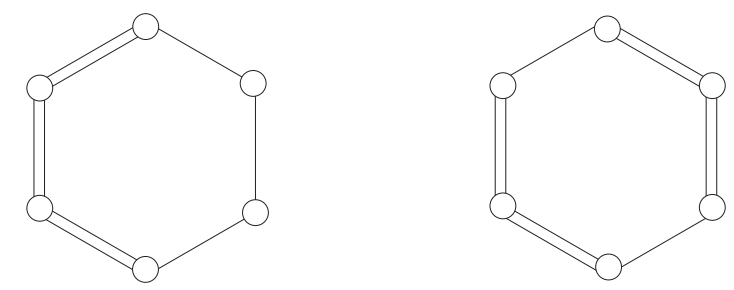

Figure 5.3. Forbidden configurations of 6-circuits in minimum counterexamples to SCDCC (Double edges with weight 2 and single edges with weight 1)

Lemma 5.2.7. Let $(G, C)$ the a minimum counterexample to SCDCC. If $G$ has a circuit $C_{6} \neq C$ of size six, then there are no configurations as shown in Figure 5.3.

Proof. Let $(G, C)$ be a minimum counterexample and $C_{6} \neq C$ be a circuit of size six. Let the weights of edges of $C_{6}$ defined as shown in Figure 5.3. Since $C_{6} \neq C$, let $v_{1} v_{6} \in E(G)-E(C)$ and hence $\omega\left(v_{1} v_{6}\right)=2$. So $\overline{G-v_{1} v_{6}}$ is smaller and hence has a circuit double cover containing $C$. Let $\mathscr{C}$ be a such circuit double cover with a maximum circuit chain joining $v_{1}$ and $v_{6}$.
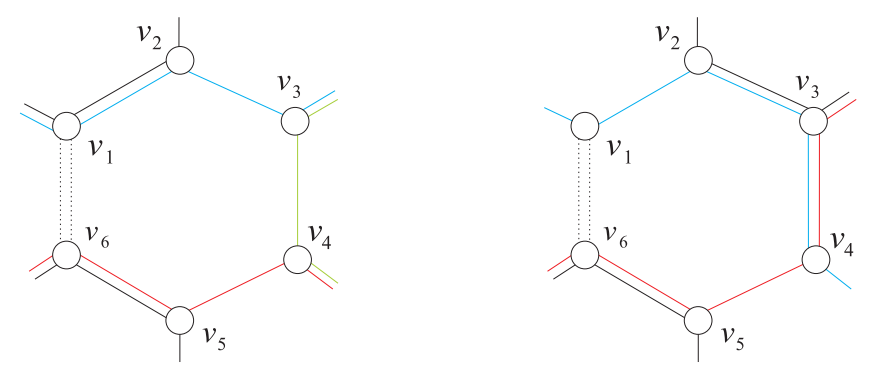

Figure 5.4. Circuit chains joining $v_{1}$ and $v_{6}$ of $C_{6}$.

If $C_{6} \cap C$ is a path of length three (the left graph in Figure 5.3), let $\mathscr{P} \subset \mathscr{C}$ be a circuit chain joining $v_{1}$ and $v_{6}$. Then $|\mathscr{P}| \leq 3$ (the red circuit $C_{r}$, the green circuit $C_{g}$ and the blue circuit $C_{b}$ form a circuit chain joining $v_{1}$ and $v_{6}$ ). By Lemma 
5.2.4, P $=\left\{C_{r}, C_{g}, C_{b}\right\}$. Note that $v_{1}$ and $v_{6}$ belong to the same component of $C_{r} \Delta C_{g} \Delta C_{b}$. Let

$$
\mathscr{C}^{\prime}:=\left(\mathscr{C}-\left\{C_{r}, C_{g}, C_{b}\right\}\right) \cup\left\{\left(C_{r} \cup C_{b}\right) \Delta C_{6}, C_{g} \Delta C_{6}\right\}
$$

Then $\mathscr{C}^{\prime}$ is a circuit double cover of $G$ containing $C$, a contradiction.

If $C_{6} \cap C$ consists of two disjoint edges (the right graph in Figure 5.3), let $\mathscr{P} \subset \mathscr{C}$ be a circuit chain joining $v_{1}$ and $v_{6}$. Note that the pattern of a circuit chain on the circuit $C_{6}$ is unique as shown in Figure 5.4 (right). So $|\mathscr{P}| \leq 2$ (the red circuit and the blue circuit), a contradiction to Lemma 5.2.4. This completes the proof.

\subsection{Circuit extension and SCDCC}

Let $G$ be a cubic graph and $C$ be a circuit of $G$. A component $B$ of $G-E(C)$ is called a Tutte bridge of $C$. A vertice in $B \cap C$ is called an attachment of $B$ on $C$. A chord of $C$ is a trivial Tutte bridge. The order of $B$ is the number of vertices in $B-C$. Let $\mathscr{T}(C, G)$ be the set of all trivial Tutte-bridges (i.e., chords of $C$ ) and let $\mathscr{N}(C, G):=V(G)-V(C)$.

Lemma 5.3.1. Let $G$ be a bridgeless cubic graph and $C$ be a circuit of $G$. Assume that $D$ is an extension of $C$ in $G$ and let $G^{\prime}:=\overline{G-(E(C)-E(D))}$. Then

$$
\left|\mathscr{T}\left(D, G^{\prime}\right)\right|+\left|\mathscr{N}\left(D, G^{\prime}\right)\right|=|\mathscr{T}(C, G)|+|\mathscr{N}(C, G)|-\epsilon
$$

where $\epsilon=\epsilon_{\mathscr{T}}+\epsilon_{\mathcal{N}}$, and $\epsilon_{\mathscr{T}}:=|\mathcal{T}(C, G) \cap E(D)|$, and $\epsilon_{\mathcal{N}}$ is the number of vertices $v$ satisfying $v \in V(D)-V(C)$ and $N(v) \cap\left(V\left(G^{\prime}\right)-V(D)\right) \neq \emptyset$. 
Proof. Let $D$ be an extension of $C$ in $G$. For a trivial Tutte-bridge $B$, if $B$ remains as a chord of $D$, then $Q \in \mathscr{T}\left(D, G^{\prime}\right)$. If $B$ is not a chord of $D$, then it is contained by $D$ and hence contributes one to $\epsilon_{\mathcal{T}}$. So $D$ contains precisely $\epsilon_{\mathcal{T}}$ trivial Tutte-bridges of $C$.

Now consider a non-trivial Tutte-bridge $B$. Let $v$ be a vertex of $B$. Then $v \in \mathscr{N}(C, G)$. If $v \notin V(D)$, then $v \in \mathscr{N}\left(D, G^{\prime}\right)$. These kind vertices do not affect the value $|\mathscr{T}(C, G)|+|\mathscr{N}(C, G)|$. So suppose $v \in V(D)$. Then $v$ has at least two neighbors on $D$. Let $u$ be the third neighbor of $v$. If $u \in V(D)$, then $u v$ is a chord of $D$. Such $v$ makes $|\mathscr{N}(C, G)|$ decrease by one, but makes $|\mathscr{T}(C, G)|$ increase by one. Hence such $v$ does not change the value $|\mathscr{T}(C, G)|+|\mathscr{N}(C, G)|$. So suppose that $u \notin V(D)$, i.e., $u \in V\left(G^{\prime}\right)-V(D)$. Then $u v$ is not a chord of $D$. So $v$ makes $|\mathscr{T}(C, G)|+|\mathscr{N}(C, G)|$ decrease one. There are precisely $\epsilon_{\mathcal{N}}$ vertices in this type. So the lemma follows.

Lemma 5.3.2. Let $G$ be a bridgeless cubic graph and $C$ be a circuit of $G$. If $G$ has no a circuit double cover containing $C$, then

$$
|\mathscr{T}(C, G)|+|\mathscr{N}(C, G)| \geq 7 .
$$

Proof. It is equivalent to prove the following statement.

A bridgeless cubic graph $G$ has a circuit double cover containing of $G$ if

$$
|\mathscr{T}(C, G)|+|\mathscr{N}(C, G)| \leq 6 .
$$

Let $(G, C)$ be a minimal counterexample to $(*)$. By Lemma 5.3.1, $C$ is a stable circuit in $G$. 
(1) Every Tutte-bridge $B$ of $C$ has order either 0 or at least two.

Suppose to the contrary, let $B$ be a Tutte-bridge of $C$ of order one. Assume that $w$ is the vertex of $B$ which is not on $C$ and let $N(w)=\{x, y, z\}$. For a vertex $u$ on the circuit $C$, let $u^{+}$be the preceding neighbor of $u$ and $u^{-}$be the succeeding neighbor of $u$ in counterclockwise direction. By Theorem 5.2.5, Lemma 5.2.6 and Lemma 5.2.7, the neighbors of $x$ on $C$ are different from neighbors of $y$ and $z$, so do $y$ and $z$.

Define an auxiliary graph $A$ such that $V(A)=\left\{x^{-}, x^{+}, y^{-}, y^{+}, z^{-}, z^{+}\right\}$, and for $u, v \in V(A), u v \in E(A)$ if and only if there exists a Tutte-bridge $B^{\prime}$ of $C$ containing both $u$ and $v$. If there exists a Tutte-bridge $B^{\prime}$ joining $x^{+}$and $y^{+}$, then $C$ has an extension $D=C-\left\{x x^{+}, y y^{+}\right\}+x w y+P_{B^{\prime}}$ where $P_{B^{\prime}}$ is a path in $B^{\prime}$ joining $x^{+}$and $y^{+}$, a contradiction. Hence, for any $u, v \in\{x, y, z\}, u^{+}$and $v^{+}$belong two different components of $A$, so do $u^{-}$and $v^{-}$. It follows that $A$ has at most three edges which are joining vertices from $\left\{x^{+}, y^{+}, z^{+}\right\}$to vertices from $\left\{x^{-}, y^{-}, z^{-}\right\}$. Let $\gamma(A)$ be the number of isolated vertices of $A$. Then $\gamma(A)=6-2|E(A)|$.

For $u, v \in\{x, y, z\}$, if $u^{+}$and $v^{-}$are contained by a bridge $B^{\prime}$, by Theorem 5.2.5, Lemma 5.2.6 and Lemma 5.2.7, the order of $B^{\prime}$ is at least two. Hence

$$
\begin{aligned}
|\mathscr{T}(C, G)|+|\mathscr{N}(C, G)| & \geq 2|E(A)|+\gamma(A)+1 \\
& =2|E(A)|+6-2|E(A)|+1 \\
& =7 .
\end{aligned}
$$

It is a contradiction to $|\mathscr{T}(C, G)|+|\mathscr{N}(C, G)| \leq 6$.

(2) Every Tutte-bridge $B$ of $C$ has order either 0 or at least three. 
By (1), suppose that $C$ has a Tutte-bridge $B$ of order two and assume that $V(B-C)=\{x, y\}$. Let $N(x) \cup N(y)-\{x, y\}=\left\{x_{1}, x_{2}, x_{3}, x_{4}\right\}$. As defined above, for any $v \in\left\{x_{1}, x_{2}, x_{3}, x_{4}\right\}$, the preceding neighbor and succeeding neighbor of $v_{i}$ on $C$ is $v_{i}^{+}$and $v_{i}^{-}$in counterclockwise direction. Now, construct an auxiliary graph $A$ again: $V(A)=\cup_{i=1}^{4} N\left(x_{i}\right) \cap V(C)$ and, for any $u, v \in V(A), u v \in E(A)$ if and only if there is a Tutte-bridge containing both $u$ and $v$. If there is a Tutte-bridge $B^{\prime}$ which contains $u^{+}$and $v^{+}$where $u, v \in\left\{x_{1}, x_{2}, x_{3}, x_{4}\right\}$, then $C-\left\{u^{+} u, v^{+} v\right\}+u x y v+P_{u^{+} v^{+}}$ is an extension, a contradiction. It follows that each component of $A$ is a $K_{2}$ and hence $|E(A)| \leq 4$. Let $\gamma(A)$ be the number of isolated vertices of $A$. Then $\gamma(A)=8-2|E(A)|$.

For $u, v \in\left\{x_{1}, x_{2}, x_{3}, x_{4}\right\}$, if $u^{+}$and $v^{-}$are contained by a bridge $B^{\prime}$, by Theorem 5.2.5, Lemmas 5.2.6 and 5.2.7, $B^{\prime}$ is not trivial. Then by (1), the order of $B^{\prime}$ is at least two. Hence

$$
\begin{aligned}
|\mathscr{T}(C, G)|+|\mathscr{N}(C, G)| & \geq 2|E(A)|+\gamma(A)+1 \\
& =2|E(A)|+8-2|E(A)|+1 \\
& =9 .
\end{aligned}
$$

It contradicts $|\mathscr{T}(C, G)|+|\mathscr{N}(C, G)| \leq 6$.

By (1) and (2), every non-trivial Tutte-bridge of $C$ has order at least three. By Theorem 4.1.7, $C$ has at least two odd Tutte-bridges and there attachments should be separated by other Tutte-bridges. It follows that $|\mathcal{T}(C, G)|+|\mathcal{N}(C, G)|>6$, also a contradiction. This completes the proof. 


\subsection{Proof of Theorem 5.1.6}

Lemma 5.4.1. Let $G$ be a cubic graph with a circuit $C$ with $|\mathscr{T}(C, G)|+|\mathscr{N}(C, G)| \leq$ 8. If $C$ has at most one non-trivial Tutte-bridge B, then $G$ has a circuit double cover containing $C$.

Proof. Let $B$ be the only non-trivial Tutte-bridge of $C$. Let $(G, C)$ be a counterexample such that:

(1) $|\mathscr{T}(C, G)|+|\mathscr{N}(C, G)|$ is smallest;

(2) subject to (1), $|V(G)|$ is smallest.

By Theorem 4.1.6, $C$ has an extension $D$ in $G$. Let $G^{\prime}:=\overline{G-(E(C)-E(D))}$. If $D$ does not pass $B$, then $\left|\mathscr{T}\left(D, G^{\prime}\right)\right|<|\mathscr{T}(C, G)|$ and $\left|\mathscr{N}\left(D, G^{\prime}\right)\right|=\left|\mathscr{N}\left(C, G^{\prime}\right)\right|$. It follows that $G^{\prime}$ has a circuit double cover $\mathscr{C}$ containing $D$. Then $G$ has a circuit double cover $(\mathscr{C}-\{D\}) \cup\{D \triangle C, C\}$, a contradiction. So assume that $D$ passes $B$.

If $D$ has at least two nontrivial Tutte-bridges, then $\epsilon_{\mathscr{N}} \geq 2$. Then $\left|\mathscr{T}\left(D, G^{\prime}\right)\right|+$ $\left|\mathscr{N}\left(D, G^{\prime}\right)\right| \leq 6$. By Lemma 5.3.1, $G^{\prime}$ has a circuit double cover containing $D$. Hence $G$ has a circuit double cover containing $C$, a contradiction.

So $D$ has at most one non-trivial Tutte-bridge. Note that $\left|V\left(G^{\prime}\right)\right|<|V(G)|$. So $G^{\prime}$ has a circuit double cover containing $D$, also a contradiction. This completes the proof.

Now we are going to prove Theorem 5.1.6.

Proof of Theorem 5.1.6. Let $B$ be a Tutte-bridge of $C$ of order at most 4 . 
Let $G_{1}$ be a subgraph of $G$ consisting of $B$ and $C$ together all chords of $C$. Let $G_{2}$ be a subgraph of $G$ consisting of $C$ and all other non-trivial Tutte-bridges. So $G=G_{1} \cup G_{2}$.

By Theorem 5.1.5, $G_{1}$ has a circuit double cover $\mathscr{C}_{1}$ containing $C$.

Now, consider $G_{2}$. Note that $\mathscr{T}\left(C, G_{2}\right)=\emptyset$. If $\left|\mathscr{N}\left(C, G_{2}\right)\right| \leq 6$, then Lemma 5.3.2 implies that $G_{2}$ has a circuit double cover $\mathscr{C}_{2}$ containing $C$. If $\left|\mathscr{N}\left(C, G_{2}\right)\right|=7$, then $B$ does not exist ( $C$ has only one non-trivial Tutte-bridge). Lemma 5.4.1 implies that $G_{2}$ has a circuit double cover $\mathscr{C}_{2}$ containing $C$.

So let $\mathscr{C}:=\left(\mathscr{C}_{1}-\{C\}\right) \cup\left(\mathscr{C}_{2}-\{C\}\right)$. It is easily check that $\mathscr{C}$ is a circuit double cover of $G$. This completes the proof.

Remark. Let $G$ be a cubic graph with a dominating circuit $C$. The strong circuit double cover conjecture claims that $G$ has a circuit double cover containing $C$. The following is a weak conjecture.

Conjecture 5.4.2. Let $G$ be a cubic graph with a chordless dominating circuit $C$. Then $G$ has a circuit double cover. 


\section{Bibliography}

[1] M. Abreu, R.E.L. Aldred, M. Funk, B. Jackson, D. Labbate and J. Sheehan, Graphs and digraphs with all 2-factors isomorphic, J. Combin. Theory B 92 (2004) 398-404.

[2] P. Adams, M. Mahdian and E.S. Mahmoodian, On the forced mathching numbers of bipartite graphs, Discrete Math. 281 (2004) 1-12.

[3] B. Alspach, L. Goddyn and C.-Q. Zhang, Graphs with the circuit cover property, Trans. Amer. Math. Soc. 344 (1994) 131-154.

[4] B. Alspach and C. Godsil, Unsolved problems, in Cycles in Graphs, Ann. Discrete Math. 27, Amsterdam, North-Holland, 1985, pp. 461-467.

[5] B. Alspach and C.-Q. Zhang, Cycle covers of cubic multigraphs, Discrete Math. 111 (1993) 11-17.

[6] G. Brinkmann, J. Goedgebeur, J. Hägglund and K. Markström, Generation and properties of snarks, preprint.

[7] M. Chan, A survey of the cycle double cover conjecture, preprint.

[8] J. Cutler and R. Häggkvist, Cycle double covers of graphs with disconnected frames, Research report 6, Department of Mathematics, Umeå University (2004). 
[9] J. Edmonds, Maximum matching and a polyhedron with (0,1)-vertices, J. Res. Nat. Bur. Standards B, 69 (1965) 125-130.

[10] L. Esperet, F. Kardos, A. King, D. Král and S. Norine, Exponentially many perfect matchings in cubic graphs, Adv. Math. 227 (2011) 1646-1664.

[11] S. Fiorini and R.J. Wilson, Edge colourings of graphs, in: L.W. Beineke, R.J. Wilson (Eds.), Selected Topics in Graph Theory, Academic Press, London, 1978, pp. 103-126.

[12] H. Fleischner, Eulerian Graph, in Selected Topics in Graph Theory (2) (L.W. Beineke and R.J. Wilson, eds.), Academic Press, London, 1983, pp. 17-53.

[13] H. Fleischner, Cycle decompositions, 2-coverings, removable cycles and the fourcolor disease, in Progress in Graph Theory (J.A. Bondy and U.S.R. Murty, eds.), Academic Press, New York, 1984, pp. 233-246.

[14] H. Fleischner, Proof of the strong 2-cover conjecture for planar graphs, J. Combin. Theory Ser. B 40 (1986) 229-230.

[15] H. Fleischner, Communication with C.-Q. Zhang at Cycle Double Cover Conjecture Workshop, Barbados, February 25-March 4, 1990.

[16] H. Fleischner, Uniqueness of maximal dominating cycles in 3-regular graphs and Hamiltonian cycles in 4-regular graphs, J. Graph Theory 18 (1994) 449-459.

[17] H. Fleischner, R. Häggkvist, Circuit double covers in special types of cubic graphs, Discrete Math. 309 (2009) 5724-5728.

[18] T. Fowler, Unique Coloring of Planar Graphs, Ph.D. Thesis, Georgia Tech., 1998.

[19] M. Funka, B. Jacksonb, D. Labbatea and J. Sheehan, 2-Factor hamiltonian graphs, J. Combin. Theory B 87 (2003) 138-144

[20] L.A. Goddyn, Cycle Covers of Graphs, Ph.D Thesis, University of Waterloo, 1988. 
[21] L.A. Goddyn, Cycle double covers of graphs with Hamilton paths, J. Combin. Theory Ser. B 46 (1989) 253-254.

[22] R. Häggkvist and K. Markström, Cycle double covers and spanning minors I, J. Combin. Theory Ser. B 96 (2006) 183-206.

[23] R. Häggkvist and K. Markström, Cycle double covers and spanning minors II, Discrete Math. 306 (2006) 726-778.

[24] R. Häggkvist and S. McGuinness, Double covers of cubic graphs with oddness 4, J. Combin. Theory Ser. B 93 (2005) 251-277.

[25] J. Hägglund, K. Markström, On stable cycles and cycle double covers of graphs with large circumference, Discrete Math., In press.

[26] A. Huck, On cycle-double covers of graphs of small oddness, Discrete Math. 229 (2001) 125-165.

[27] A. Huck and M. Kochol, Five cycle double covers of some cubic graphs, $J$. Combin. Theory Ser. B 64 (1995) 119-125.

[28] A. Hoffmann-Ostenhof, Nowhere-Zero Flows and Structures in Cubic Graphs, $\mathrm{PhD}$ thesis.

[29] A. Itai and M. Rodeh, Covering a graph by circuits, in: Automata, Languages and Programming, Lecture Notes Comput. Sci. 62, Springer-Verlag, Berlin, 1978, pp. 289-299.

[30] B. Jackson, On circuit covers, circuit decompositions and Euler tours of graphs, in: Surveys in Combinatorics, (Keele ed.), London Math. Soc. Lecture Notes Ser. 187, Cambridge Univ. Press, Cambridge, 1993, pp. 191-210.

[31] F. Jaeger, A survey of the cycle double cover conjecture, in Cycles in Graphs (B. Alspach and C. Godsil, eds.), Ann. Discrete Math. 27 (1985) pp. 1-12.

[32] J. Kahn, N. Robertson and P.D. Seymour, etc., Communication at Bellcore, 1987 
[33] M. Kochol, Stable dominating circuits in snarks, Discrete Math. 233 (2001) $247-256$.

[34] A. Kotzig, On the theory of finite graphs with a linear factor, I, Mat.-Fyz. Časopis Slovensk. Akad. Vied 9 (1959) 73-91 (Slovak).

[35] A. Kotzig, Hamilton graphs and Hamilton circuits, in: Theory of Graphs and its Applications, Proceedings of the Symposium of Smolenice, 1963, Publ. House Czechoslovak Acad. Sci., Prague, 1964, pp. 63-82.

[36] A. Kotzig and J. Labelle, Strongly hamiltonian graphs, Utilitas Math. 14 (1978) 99-116.

[37] H.-J. Lai and C.-Q. Zhang, Hamilton weights and Petersen minors. J. Graph Theory 38 (2001), no. 4, 197-219.

[38] L. Lovász and M.D. Plummer, Matching Theory, Ann. Discrete Math. 29, Amsterdam, 1986.

[39] Z. Miao, D. Ye and C.-Q. Zhang, Circuit extension and circuit double cover of graphs, submitted.

[40] C. St. J. A. Nash-Williams, Edge disjoint spanning trees of finite graphs, J. London Math. Soc. 36 (1961) 445-450.

[41] J.P.C. Peterson, Die theorie der regularen graphs, Acta Mathematica, 15 (1891) 193-220.

[42] J. Plensník, Connectivity of regular graphs and the existence of 1-factors, Mat. Časopis Sloven. Akad. Vied 22 (1972) 310-318.

[43] P.D. Seymour, Sums of circuits, in: Graph Theory and Related Topics (J.A. Bondy and U.S.R. Murty, eds.), Academic Press, New York, 1979, pp. 342-355.

[44] P.D. Seymour, Communication with C.-Q. Zhang at Cycle Double Cover Conjecture Workshop, Barbados, February 25-March 4, 1990.

[45] P. D. Seymour, Personal communication with C.-Q. Zhang, 2012. 
[46] G. Szekeres, Polyhedral decompositions of cubic graphs, Bull. Austral. Math. Soc. 8 (1973) 367-387.

[47] M. Tarsi, Semi-duality and the cycle double cover conjecture, J. Combin. Theory Ser. B 41 (1986) 332-340.

[48] A. Thomason, Hamiltonian Cycles and uniquely edge colorable graphs, Ann. Discrete Math. 3 (1978) 259-268.

[49] W. T. Tutte, On the problem of decomposing a graph into $n$ connected factors, J. London Math. Soc. 36 (1961) 221-230.

[50] W. T. Tutte, Personal correspondence to H. Fleischner, July 22, 1987.

[51] D. Ye and C.-Q. Zhang, Cycle double covers and the semi-kotzig frame, European J. Combin. 33 (4) (2012) 624-631.

[52] C.-Q. Zhang, Hamiltonian weights and unique 3-edge-colorings of cubic graphs, J. Graph Theory 20 (1995) 91-99.

[53] C.-Q. Zhang, Nowhere-zero 4-flows and cycle double covers, Discrete Math. 154 (1996) 245-253.

[54] C.-Q. Zhang, Integer Flows and Cycle Covers of Graphs, Marcel Dekker, NewYork, 1997.

[55] C.-Q. Zhang, On strong 1-factors and Hamilton weights of cubic graphs, Discrete Math. 230 (2001) 143-148.

[56] C.-Q. Zhang, Circuit Double Covers of Graphs, Cambridge University Press, London, 2012.

[57] X. Zhang and C.-Q. Zhang, Kotzig frames and circuit double covers, Discrete Math. 312 (1) (2012) 174-180. 